\title{
Accretion-driven gravitational radiation from nonrotating compact objects: Infalling quadrupolar shells.
}

\author{
Alessandro Nagar, ${ }^{1,2}$ Guillermo Díaz, ${ }^{1}$ José A. Pons, ${ }^{3}$ and José A. Font ${ }^{1}$ \\ ${ }^{1}$ Departament d'Astronomia $i$ Astrofísica, Universitat de València, \\ Edifici d'Investigació, Dr. Moliner 50, 46100 Burjassot (Valencia), Spain \\ ${ }^{2}$ Dipartimento di Fisica, Università di Parma, Parco Area delle Scienze 7A, 43100 Parma, Italy \\ ${ }^{3}$ Departament de Física Aplicada, Universitat d'Alacant, Ap. Correus 99, 03080 Alacant, Spain
}

(Dated: April 18, 2018)

\begin{abstract}
This paper reports results from numerical simulations of the gravitational radiation emitted from non-rotating compact objects (both neutron stars and Schwarzschild black holes) as a result of the accretion of matter. We adopt a hybrid procedure in which we evolve numerically, and assuming axisymmetry, the linearized equations describing metric and fluid perturbations coupled to a fully nonlinear hydrodynamics code that calculates the motion of the accreting matter. The initial matter distribution, which is initially at rest, is shaped in the form of extended quadrupolar shells of either dust or obeying a perfect fluid equation of state. Self-gravity of the accreting layers of fluid is neglected, as well as radiation reaction effects. We use this idealized setup in order to understand the qualitative features appearing in the energy spectrum of the gravitational wave emission from compact stars or black holes, subject to accretion processes involving extended objects. A comparison for the case of point-like particles falling radially onto black holes is also provided. Our results show that, when the central object is a black hole, the spectrum is far from having only one clear, monochromatic peak at the frequency of the fundamental quasi-normal mode. On the contrary, it shows a complex pattern, with distinctive interference fringes produced by the interaction between the infalling matter and the underlying perturbed spacetime, in close agreement with results for point-like particles. Remarkably, most of the energy is emitted at frequencies lower than that of the fundamental mode of the black hole. Similar results are obtained for extended shells accreting onto neutron stars, but in this case the contribution of the stellar fundamental mode stands clearly in the energy spectrum. Our analysis illustrates that the gravitational wave signal driven by accretion onto compact objects is influenced more by the details and dynamics of the process, and the external distribution of matter, than by the quasi-normal mode structure of the central object. The gravitational waveforms from such accretion events appear to be much more complex than former simplified assumptions predicted.
\end{abstract}

PACS numbers: $04.30 . \mathrm{Db}$, 04.40.Dg, 95.30.Lz, 98.62.Mw

\section{INTRODUCTION}

Most of the different astrophysical scenarios suggested as potential sources of gravitational radiation have in common the presence of compact objects, such as neutron stars, strange stars or black holes. The coalescence of a binary system formed by two black holes, two neutron stars or one black hole and one neutron star is the main target of the ground based interferometers [Laser Interferometer Gravitational Wave Observer (LIGO), VIRGO), but other possibilities such as galactic supernovae are also worth exploring, especially in anticipation of the capabilities of future detectors. Isolated black holes, in particular, are reckoned to be characterized by a unique emission pattern known as quasi-normal mode (QNM) ringing-rapidly damped sinusoidal modes. This signal has been studied extensively using perturbation theory and frequency-domain techniques for most classes of black hole solutions (see e.g. 1, 2] and references therein). The detection of such QNM signals depends strongly on the luminosity of the source or, in other words, on how strongly the black hole is excited, but also on the knowledge of the power spectrum. It is also well known that a relativistic star has a very rich non-radial oscillation spectrum, and it can emit gravitational waves through QNM ringing (see e.g. 2] and references therein). Therefore, another plausible source of gravitational radiation involves the rapid accretion of large clumps of matter onto a compact object (either a neutron star or a black hole), a recurrent and ubiquitous phenomenon in relativistic astrophysics. Accretion is expected to happen following the gravitational collapse of the core of a massive star, once a neutron star has already been formed. Part of the remaining stellar material, which has not been expelled by the shock driving the supernova explosion, may fall back onto the neutron star, until a critical mass is exceeded and the star collapses to a black hole. Some more material may in turn form a long-lived, centrifugally supported torus or disk if the collapsing star had initially some amount of rotation.

Semi-analytical studies of extended objects (shells or blobs of dust) falling isotropically onto a black hole are available in the literature [3, 4, 5, 6, 7, 8]. These studies shed the first light in understanding the modification of the gravitational wave (GW) emission pattern of the black hole due to the presence of matter. Collectively, these works showed that for a fixed amount of infalling mass $m$, the energy released in gravitational radiation is 
reduced compared to the value of the point-particle limit ( $E \sim 0.01 \mathrm{~m}^{2} / M[9], M$ being the mass of the black hole). This reduction is interpreted as due to cancellations of the emission from distinct parts of the extended object. Such conclusions were later confirmed by Papadopoulos and Font [10], who performed numerical simulations of the gravitational radiation emitted during the accretion process of an extended object onto a black hole. In [10] the first-order deviations from the exact black hole geometry were approximated by the Teukolsky equation 11] for Schwarzschild black holes, i.e. the inhomogeneous Bardeen-Press equation [12], including curvature perturbations induced by matter sources, whose nonlinear evolution was integrated using a hydrodynamics code. This was the first numerical study in the time-domain of the gravitational radiation emitted by extended objects accreted by black holes, and showed the gradual excitation of the black hole fundamental QNM frequency by sufficiently compact shells. In the thin shell limit, the energy asymptotes to a finite value, which is about a third of the point-particle limit.

Correspondingly, linear perturbation studies in the time domain of neutron star spacetimes, aimed at addressing QNM excitation, have also received considerable attention in the literature (see e.g. 2] and references therein). The oscillation properties are obtained from the analysis of non-radial stellar perturbations. For non-rotating stars with polytropic equations of state, the spectrum naturally splits into an axial (or odd-parity) and a polar (or even-parity) part, according to the terminology used to address the behaviour of the perturbation equations under parity transformations. The axial part of the spectrum includes only gravitational modes, the so called $w$ modes, which are purely relativistic, being absent in Newtonian gravity [13]. The polar part, on the other hand, contains $w$ modes as well as fluid modes. The excitation of axial parity modes was analyzed by Andersson and Kokkotas [14], by sending pulses of gravitational waves to the neutron star and studying its response, and by Ferrari and Kokkotas [15], by scattering of point-like particles. The excitation of polar modes was first investigated by Allen et al. [16], also as a scattering problem using gravitational wave pulses. The same approach was later followed by Ruoff and co-workers in [17, 18], using both Gaussian pulses and point-particle scattering. A framework for constructing initial data sets for perturbations was developed in 19] and applied to study neutron star collisions in the close limit approximation in Ref. [20].

Alternately, in a series of papers Seidel and co-workers computed the gravitational radiation emanating from slightly non-spherical stellar core collapse, in the axial 21] as well as in the polar case 22], and the waveforms associated with the formation of neutron stars. The zerothorder solution was a spherical collapsing star, whose dynamics was computed by solving the coupled system of Einstein and hydrodynamics equations using the Lagrangian May-White approach. The GWs were extracted using perturbation theory on the spherical background within the Gerlach-Sengupta 23] formalism. More recently, Harada et al. 24 have reexamined the axial part of this problem, using null coordinates (HernandezMisner) and a gauge invariant and coordinate independent perturbative formalism developed by Martín-García and Gundlach [25, 26, 27]. Within this approach Harada et al. 24] have been able to follow the spherical collapse of both, supermassive stars and neutron stars, until a black hole forms, computing the GWs that are emitted in the process. Full numerical relativity computations of QNM ringing in spherical gravitational collapse using the characteristic formulation of general relativity are also reported in [28]. Within the same formalism, the imprints of fluid accretion on the emitted GWs from a black hole were studied in [29], finding the familiar damped-oscillatory GW decay, but both decay rate and frequencies being modulated by the mass accretion rate.

In this paper, we analyze the similarities and differences in the gravitational wave emission pattern from black holes and neutron stars as a result of the radial accretion of matter. A detailed realistic modelization of the gravitational emission from accretion flows would require of three-dimensional (magneto-)hydrodynamical simulations in general relativity, coupled to radiation transport and diffusive processes. However, some preliminary steps can be taken to understand the underlying basic physics in a qualitative way before getting engaged in large scale computational efforts. Our numerical procedure lies, hence, in the borderline of full numerical relativity and perturbation theory. As in [10], the accreting matter is evolved in a curved static background by solving the nonlinear hydrodynamics equations. The response of the compact object to the infalling matter, which triggers the emission of gravitational radiation, is computed using perturbation theory. More precisely, we use the gauge invariant formalism of [26] and study the excitation of QNMs of both Schwarzschild black holes and neutron stars by numerically solving in the time domain the even-parity perturbation equations with matter sources. For the case of black holes, these equations reduce to the inhomogeneous Zerilli-Moncrief [30, 31] equation. One key assumption of our approach is that the mass of the accreting fluid is much smaller than the mass of the central compact object. Fluid self-gravity and radiation reaction effects are also neglected; i.e., we ignore the first-order metric corrections to the fluid equations of motion. The first approximation (no self-gravity) is in general valid for fluid motions in the vicinity of the compact object, where tidal forces dominate over the fluid self-gravity. The second approximation (no radiation reaction) is valid as long as the energy in the form of gravitational radiation is much smaller than the kinetic or internal energy of the fluid. Our procedure follows then the same hybrid approach previously adopted in [10], but departs from it in the formalism. We anticipate that, for a given numerical resolution and numerical scheme, the use of the Zerilli-Moncrief equation results in im- 
proved long-term numerical stability as compared to the Bardeen-Press equation employed by [10], allowing for an accurate computation of late time features of the GW signal (namely tails). The results reported in the present investigation are further restricted to the case of radially accreting shells of either dust or obeying a perfect fluid equation of state (EOS), where the mass density profile is shaped in the form of quadrupolar shells of Gaussian radial extent. In this respect this work can be considered as a necessary assessment of our numerical approach, in anticipation of the study of more interesting scenarios, namely the excitation of QNMs from perfect fluid thick accretion tori orbiting around compact objects, which will be presented elsewhere [32].

The paper is organized as follows: Section $\amalg$ describes in some detail the theoretical framework adopted, namely the construction of the unperturbed stellar models, the general relativistic hydrodynamics equations, the perturbation equations for neutron stars and black holes, and the generation of time-symmetric initial data to describe the accreting shells. The numerical methods used for both the hydrodynamics equations and the perturbation equations are outlined in Sec. III Section IV is devoted to presenting the main results of our investigation, splitting the description of the black hole and neutron star cases into two separate subsections. Finally, Sec. D summarizes the main conclusions of this work and outlines future directions in this research. Appendix $\$$ contains technical information regarding the general form of the source term for the inhomogeneous Zerilli-Moncrief equation, and Appendix B presents a comparison with pointlike particles falling onto black holes. We use units such that $c=G=1$.

\section{THEORETICAL FRAMEWORK}

\section{A. Unperturbed stellar models}

The background metric of a non-rotating, spherically symmetric star is given by the line element

$$
d s^{2}=-e^{2 a} d t^{2}+e^{2 b} d r^{2}+r^{2}\left(d \vartheta^{2}+\sin ^{2} \vartheta d \varphi^{2}\right),
$$

where $a$ and $b$ are functions of the radial coordinate $r$ only. Assuming the star is a perfect fluid whose energy momentum tensor reads

$$
T_{\mu \nu}=(\epsilon+p) u_{\mu} u_{\nu}+p g_{\mu \nu},
$$

with $p$ denoting the pressure, $\epsilon$ the total energy density, and $u^{\mu}$ the fluid 4 -velocity, the Einstein's equations become the Tolman-Oppenheimer-Volkoff (TOV) equations
TABLE I: Frequencies of the first fluid and gravitational modes for the two neutron star models considered.

\begin{tabular}{ccc}
\hline \hline Mode & $\mathrm{A}[\mathrm{kHz}]$ & $\mathrm{B}[\mathrm{kHz}]$ \\
\hline$f$ & 2.584 & 1.666 \\
$p_{1}$ & 3.948 & 4.045 \\
$w_{1}$ & 11.609 & 10.380 \\
$w_{2}$ & 20.197 & 18.429 \\
\hline \hline
\end{tabular}

of hydrostatic equilibrium:

$$
\begin{aligned}
\frac{d m}{d r} & =4 \pi r^{2} \epsilon, \\
\frac{d a}{d r} & =\frac{\left(m+4 \pi r^{3} p\right)}{\left(r^{2}-2 m r\right)}, \\
\frac{d p}{d r} & =-(\epsilon+p) \frac{d a}{d r},
\end{aligned}
$$

where $m(r)$ is the gravitational mass enclosed in a sphere of radius $r$. The surface of the star $r=R$ is determined by the condition $p=0$. At the exterior the geometry reduces to the Schwarzschild solution. The above system of ordinary differential equations (ODEs) can be integrated once the EOS is chosen and a value of the central energy density $\epsilon_{c}$ is specified. We further assume the fluid to be isentropic, so that we adopt a one-parameter EOS, $p=p(\epsilon)$, in polytropic form $p=K \epsilon^{\Gamma}$, where $\Gamma$ is the adiabatic exponent.

For the calculations we discuss in this work, we will consider two stellar models with the same gravitational mass, $M=1.4 M_{\odot} \approx 2.067 \mathrm{~km}$, and the same adiabatic exponent $\Gamma=2$, one model being more compact than the other. These two models are meant to bracket the interval of possible radii of realistic neutron stars. The more compact model (A) has $\epsilon_{c}=2.455 \times 10^{15} \mathrm{~g} / \mathrm{cm}^{3}$ and $K=122.25 \mathrm{~km}^{2}$, so that the radius is $R=9.80 \mathrm{~km}$. The less compact model (B) has $\epsilon_{c}=0.92 \times 10^{15} \mathrm{~g} / \mathrm{cm}^{3}$ and $K=180 \mathrm{~km}^{2}$ which leads to $R=13.44 \mathrm{~km}$.

Our analysis is further restricted to polar perturbations induced by external matter flows. For the sake of reference, the first frequencies of the polar part of the spectrum of the two stellar models considered are listed in Table 【 They have been obtained using a frequencydomain code based on the classical Lindblom-Detweiler formulation of the perturbed Einstein equations [33] and described in [34].

\section{B. General relativistic hydrodynamics equations}

The motion of a fluid in a curved spacetime is governed by the local conservation laws of baryonic number and energy momentum:

$$
\nabla_{\mu} J^{\mu}=0, \quad \nabla_{\mu} t^{\mu \nu}=0,
$$


where $J^{\mu}=\rho u^{\mu}$ is the mass density current and $t^{\mu \nu}=\rho h u^{\mu} u^{\nu}+p g^{\mu \nu}$ is the stress energy tensor for a perfect fluid. In these expressions, $\rho$ is the rest-mass density, $h$ is the specific enthalpy, defined as $h=1+e+p / \rho$, and $e$ is the specific internal energy. The system of equations is closed with an $\operatorname{EOS} p=p(\rho, e)$.

The equations of general relativistic hydrodynamics are a system of hyperbolic equations; as shown by 35], it can be explicitly written as a system of conservation laws. This is accomplished by defining quantities which are directly measured by Eulerian observers, i.e. the rest mass density $D=\rho W$, the momentum density in the $j$ direction $S_{j}=\rho h W^{2} v_{j}$ and the total energy density $E=\rho h W^{2}-p$. In these definitions, $W$ stands for the Lorentz factor, which satisfies $W=\left(1-v^{2}\right)^{-1 / 2}$, with $v^{2}=\gamma_{i j} v^{i} v^{j}$. Here, $v^{i}$ is the 3-velocity of the fluid, defined as $v^{i}=u^{i} / W+\beta^{i} / \alpha$, where $\alpha$ and $\beta^{i}$ are the spacetime lapse function and shift vector, respectively, and $\gamma_{i j}$ are the spatial components of the spacetime metric where the fluid evolves. For a generic spacetime, the system of equations we solve reads 35

$$
\frac{1}{\sqrt{-g}}\left(\frac{\partial \sqrt{\gamma} \mathbf{U}(\mathbf{w})}{\partial t}+\frac{\partial \sqrt{-g} \mathbf{F}^{i}(\mathbf{w})}{\partial x^{i}}\right)=\mathbf{S}(\mathbf{w}),
$$

where $g=\operatorname{det}\left(g_{\mu \nu}\right), \mathbf{U}(\mathbf{w})=\left(D, S_{j}, E-D\right)$, and $\mathbf{w}=\left(\rho, v_{i}, e\right)$ is the vector of primitive variables. The expressions for the flux and source vectors, $\mathbf{F}^{\mathbf{i}}(\mathbf{w})$ and $\mathbf{S}(\mathbf{w})$, can be found in Ref. 35. We note that in the current work we use the standard form of the Schwarzschild metric in polar-radial coordinates $(t, r, \vartheta, \varphi)$ to describe the exterior spacetime of the compact object. Hence, the above expressions are specialized accordingly.

\section{Stellar polar metric perturbations induced by hydrodynamical sources}

\section{Interior equations}

A formulation of the equations describing the polar perturbations of a star in the Regge-Wheeler gauge [36], written in a form suitable for numerical simulations in the time domain, was first discussed by [16]. An alternative derivation of the same equations, based on the linearized Arnowitt-Deser-Misner (ADM) formalism, can be found in 17]. Recently, building on the work of Gerlach and Sengupta 23], Martín-García and Gundlach have developed a gauge-invariant and coordinate-independent formalism for non-spherical perturbations of spherically symmetric spacetimes $25,26,27]$. In our work, we follow the formalism laid out in [26] specifying the equations to the case of a static spherical star and choosing the ReggeWheeler gauge [36]. The equations we obtain are then equivalent to those of Refs. [16] and [17], although different metric variables are used. Hence, for each $(l, m)$ pair, the even-parity metric perturbation $\delta g_{\mu \nu}$ is parametrized by two scalar quantities, $k$ (the perturbed 3-conformal factor) and $\chi$ (the actual gravitational wave degree of freedom), so that it reads

$$
\delta g_{\mu \nu}=\left(\begin{array}{cccc}
(\chi+k) e^{2 a} & -\psi e^{a+b} & 0 & 0 \\
-\psi e^{a+b} & (\chi+k) e^{2 b} & 0 & 0 \\
0 & 0 & k r^{2} & 0 \\
0 & 0 & 0 & k r^{2} \sin ^{2} \vartheta
\end{array}\right) Y_{\ell m}
$$

Since the background is static, $\psi$ is not an independent quantity, as it can be obtained by quadrature from $k$ and $\chi$ [25]; the relationship with the usual Regge-Wheeler variables can also be found in [25]. We note that, although the polar problem on a static star is known to have only two metric degrees of freedom inside the star and one degree of freedom outside (represented by the Zerilli-Moncrief function), we have decided to consider an additional variable inside the star, the perturbation of the relativistic enthalpy $H=\delta p /(p+\epsilon)$, as suggested by earlier studies of the subject [16, 17]. Correspondingly, at the exterior we evolve two (constrained) degrees of freedom instead of just one (see below). We notice, however, that successful evolution algorithms using the actual number of degrees of freedom have been developed in the past in more general frameworks [22].

We formulate, then, the polar perturbation problem through a couple of hyperbolic equations for $\chi$ and $H$ plus an elliptic equation, the Hamiltonian constraint, which is used to compute $k$ at every temporal slice. This permits us to obtain the frequencies of the stellar pulsation modes with an accuracy comparable to frequency domain calculations. The set of equations reads 


$$
\begin{aligned}
\chi_{, t t}-e^{2(a-b)} \chi_{, r r}=- & e^{2 a}\left\{-2\left[2 e^{2 b}\left(\frac{m}{r^{2}}+4 \pi r p\right)^{2}+8 \pi \epsilon-\frac{6 m}{r^{3}}\right](\chi+k)+\frac{\lambda-2}{r^{2}} \chi\right. \\
- & {\left.\left[4 \pi r(5 p-\epsilon)-\frac{2}{r}+10 \frac{m}{r^{2}}\right] \chi, r\right\}, } \\
H_{, t t}-c_{s}^{2} e^{2(a-b)} H_{, r r}=- & e^{2 a}\left\{\left[\frac{m}{r^{2}}\left(1+c_{s}^{2}\right)+4 \pi r p\left(1-2 c_{s}^{2}\right)+\left(4 \pi r \epsilon-\frac{2}{r}\right) c_{s}^{2}\right] H_{, r}\right. \\
- & {\left[4 \pi(p+\epsilon)\left(3 c_{s}^{2}+1\right)-c_{s}^{2} \frac{\lambda}{r^{2}}\right] H+\frac{1}{2}\left(\frac{m}{r^{2}}+4 \pi p r\right)\left(1-c_{s}^{2}\right)\left(\chi, r-k_{, r}\right) } \\
+ & {\left.\left[\frac{2\left(m+4 \pi p r^{3}\right)^{2}}{r^{3}(r-2 m)}-4 \pi(3 p+\epsilon) c_{s}^{2}\right](\chi+k)\right\}, } \\
e^{-2 b} k_{, r r}-\left(\frac{\lambda}{r^{2}}-8 \pi \epsilon\right) k+ & \left(8 \pi \epsilon-\frac{\lambda+2}{2 r^{2}}\right) \chi-\frac{e^{-2 b}}{r} \chi, r+\left(\frac{2}{r}-\frac{3 m}{r^{2}}-4 \pi \epsilon r\right) k_{, r}+\frac{8 \pi(p+\epsilon)}{c_{s}^{2}} H=0,
\end{aligned}
$$

where $\lambda=l(l+1)$ and $c_{s}^{2}=\partial p / \partial \epsilon$ is the sound speed. At the star surface $\epsilon, p$ and $c_{s}^{2}$ vanish, and $m(R)=M$; thereby the evolution equation for $H$ reduces to the ODE

$$
\begin{array}{r}
H_{, t t}=-\frac{M(R-2 M)}{R^{3}}\left[\begin{array}{lr}
H_{, r}+\frac{1}{2} & \left(\chi_{, r}-k_{, r}\right)
\end{array}\right] \\
-\frac{2 M^{2}}{R^{4}}(\chi+k) .
\end{array}
$$

Since we are setting $\Gamma=2$, the term proportional to $H$ in the Hamiltonian constraint (11) is regular at $r=R$.

\section{Exterior equations with a general source term}

The exterior equations in vacuum are readily obtained from the interior equations setting $\rho=p=0$ and $m=M$. As we have seen, the equations for the induced metric perturbations are basically wave equations with potentials. The presence of an extended object outside the star or black hole reflects in the fact that these equations are not homogeneous anymore, but they contain source terms involving the stress-energy tensor of the external fluid, $t_{\mu \nu}$. Whereas for point-like particles these source terms can be explicitly computed by analytic techniques [18, 30], in the case of extended objects they involve steps that cannot be managed analytically [10]. To the best of our knowledge, an explicit expression of the source terms for a general stress energy tensor has not yet been reported in the literature.

Following the notation of Ref. [25], the (gaugeinvariant) decomposition of $t_{\mu \nu}$ in polar spherical har- monics reads

$$
\begin{aligned}
t_{\mu \nu} & =\sum_{l=0}^{\infty} \sum_{m=-l}^{l} t_{\mu \nu}^{\ell m} \\
& =\sum_{l=0}^{\infty} \sum_{m=-l}^{l}\left(\begin{array}{cc}
T_{A B}^{\ell m} Y^{\ell m} & T_{A}^{\ell m}\left(Y^{\ell m}\right)_{: a} \\
T_{A}^{\ell m}\left(Y^{\ell m}\right)_{: a} & r^{2} T_{3}^{\ell m} Y^{\ell m} \gamma_{a b}+T_{2}^{\ell m} Z_{a b}^{\ell m}
\end{array}\right)
\end{aligned}
$$

where the capital indexes run over the $M^{2}$ Lorentzian manifold and the lowercase indexes over the unit radius 2-sphere $S^{2}$, as the background spacetime can be written as a direct product $M^{2} \times S^{2}$. We also follow [25] for the definition of the scalar and vector spherical harmonics, $Y^{\ell m}$ and $\left(Y^{\ell m}\right)_{: a}$, respectively, and of the tensor spherical harmonic, $Z_{a b}^{\ell m} \equiv Y_{: a b}^{\ell m}+\frac{\lambda}{2} \gamma_{a b} Y^{\ell m}$. Here, the notation : $a$ stands for the covariant derivative with respect to the metric $\gamma_{a b} \equiv \operatorname{diag}\left(1, \sin ^{2} \vartheta\right)$ of $S^{2}$. In Ref. 25], the homogeneous equations of linearized nonspherical polar perturbations of a general time-dependent spherically symmetric spacetime were obtained. If we choose the background spacetime to be the Schwarzschild solution and interpret $t_{\mu \nu}$ as a source induced by a certain distribution of matter on this spacetime, one can arrive after some algebra at the polar perturbation equations of a Schwarzschild spacetime with source terms, which are given by 


$$
\begin{aligned}
& \chi_{, t t}-e^{2(a-b)} \chi_{, r r}=-e^{2 a}\left\{-\frac{2 M}{r^{3}}\left[2 e^{2 b} \frac{M}{r}-6\right](\chi+k)+\frac{\lambda-2}{r^{2}} \chi-\frac{2}{r}\left(\frac{5 M}{r}-1\right) \chi_{, r}\right\}+16 \pi S_{\chi}, \\
& e^{-2 b} k_{, r r}+\left(\frac{2}{r}-\frac{3 M}{r^{2}}\right) k_{, r}-\frac{e^{-2 b}}{r} \chi_{, r}-\frac{\lambda}{r^{2}} k-\frac{\lambda+2}{2 r^{2}} \chi+8 \pi S_{\mathcal{H}}=0
\end{aligned}
$$

The sources $S_{\chi}$ and $S_{\mathcal{H}}$ read

$$
\begin{aligned}
& S_{\chi}:=e^{2(a-b)}\left\{T_{11}^{\ell m}+\left(T_{2}^{\ell m}\right)_{, r r}-2\left(T_{1}^{\ell m}\right)_{, r}\right.+\frac{1}{r}\left(\frac{5 M}{r} e^{2 b}-3\right)\left(T_{2}^{\ell m}\right)_{, r}-\frac{2}{r}\left(\frac{3 M}{r} e^{2 b}-1\right) T_{1}^{\ell m} \\
&\left.+\frac{1}{r^{2}}\left[\frac{2 M^{2}}{r^{2}} e^{4 b}+8-\left(\frac{4 M}{r}+\frac{\lambda+8}{2}\right) e^{2 b}\right] T_{2}^{\ell m}-e^{2 b} T_{3}^{\ell m}\right\}, \\
& S_{\mathcal{H}}:=e^{-2 a} T_{00}^{\ell m} .
\end{aligned}
$$

It is known that the perturbations of the Schwarzschild spacetime are described by a hyperbolic equation for a single function, originally written in the frequency domain and in the Regge-Wheeler gauge by Zerilli [30] and later in the time domain by Moncrief [31], but adopting a gauge invariant formulation. Following the normalization convention of [17], the Zerilli-Moncrief function is related to $k$ and $\chi$ as follows [27, 31]

$$
Z=\frac{4 r^{2} e^{-2 b}}{\lambda[(\lambda-2) r+6 M]}\left[\chi+\left(\frac{\lambda}{2}+\frac{M}{r}\right) e^{2 b} k-r k_{, r}\right] .
$$

In presence of matter sources, this function is a solution of the inhomogeneous Zerilli-Moncrief equation

$$
Z_{, t t}-e^{2(a-b)} Z_{, r r}=\frac{2 M}{r^{2}} e^{2 a} Z_{, r}+V_{l} Z+S_{z}
$$

where the Zerilli potential $V_{l}$ is given by

$$
\begin{aligned}
& V_{l}=-\left(1-\frac{2 M}{r}\right) \\
& \times \frac{\lambda(\lambda-2)^{2} r^{3}+6(\lambda-2)^{2} M r^{2}+36(\lambda-2) M^{2} r+72 M^{3}}{r^{3}[(\lambda-2) r+6 M]^{2}} .
\end{aligned}
$$

The source term $S_{z}$ appearing in Eq. (19) for a general matter distribution has not been reported in the literature in a form suitable for time domain computations. Its derivation can be found in Appendix A. The final result is

$$
\begin{aligned}
& S_{z}=-\frac{16 \pi e^{2 a}}{\lambda[(\lambda-2) r+6 M]}\left\{\frac{e^{-2 a}}{(\lambda-2) r+6 M}\left[\lambda\left(6 r^{3}-16 M r^{2}\right)-r^{3} \lambda^{2}-8 r^{3}+68 M r^{2}-108 M^{2} r\right] T_{00}^{\ell m}\right.
\end{aligned}
$$

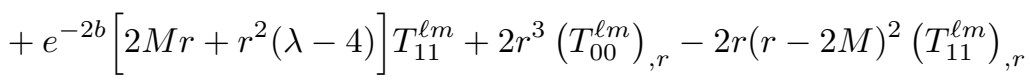

$$
\begin{aligned}
& \left.+4 \lambda(r-2 M) T_{1}^{\ell m}+\left[2 \lambda\left(1-\frac{3 M}{r}\right)-\lambda^{2}\right] T_{2}^{\ell m}+4 r(r-2 M) T_{3}^{\ell m}\right\} \text {. }
\end{aligned}
$$

In the case of a point particle, this general source term reduces to that given in [18]. 
For any multipole $(l, m)$ the radiated energy is computed from the Zerilli function. It is given by

$$
\begin{aligned}
E^{\ell m} & =\int_{-\infty}^{\infty}\left(\frac{d E}{d t}\right)^{\ell m} d t \\
& =\frac{1}{64 \pi} \frac{(l+2) !}{(l-2) !} \int_{-\infty}^{\infty}\left|Z_{, t}^{\ell m}\right|^{2} d t .
\end{aligned}
$$

By defining the Fourier transform $\tilde{Z}$ of $Z \equiv Z^{\ell m}$ as

$$
\tilde{Z}(\omega, r)=\int_{-\infty}^{\infty} e^{-i \omega t} Z(t, r) d t
$$

we obtain the energy as follows:

$$
\begin{aligned}
E^{\ell m} & =\int_{0}^{\infty}\left(\frac{d E}{d \omega}\right)^{\ell m} d \omega \\
& =\frac{1}{64 \pi^{2}} \frac{(l+2) !}{(l-2) !} \int_{0}^{\infty} \omega^{2}|\tilde{Z}(\omega, r)|^{2} d \omega .
\end{aligned}
$$

\section{Black hole polar metric perturbations induced by hydrodynamical sources}

When the neutron star is replaced by a Schwarzschild black hole, the polar perturbation problem becomes much simpler, since one only needs to solve the inhomogeneous Zerilli-Moncrief equation. As mentioned in the Introduction, in order to draw a comparison with the neutron star case, we consider the black hole case discussed in Ref. [10], but solving Eq. (19) instead of the inhomogeneous Bardeen-Press (BP) equation. The underlying motivation behind this choice is the possibility of performing long-term stable evolutions that allow for the extraction of late time features (radiative power-law tails) in the GW signals.

Working with the same numerical method, this result seems to be unreachable with the BP equation, because this equation is intrinsically unstable. To make the argument clearer, let us recall that this equation, written using the Regge-Wheeler tortoise coordinate $r^{*}$ 36],

$$
r^{*}=r+2 M \log \left(\frac{r}{2 M}-1\right)
$$

reads

$$
\begin{aligned}
Y_{, t t}-Y_{, r^{*} r^{*}} & -\frac{4(r-3 M)}{r^{2}}\left(Y_{, t}+Y_{, r^{*}}\right) \\
& =-\frac{\Delta}{r^{4}}\left[\frac{6 M}{r}+(l+2)(l-1)\right] Y+\frac{8 \pi \Delta}{r} \mathcal{T},
\end{aligned}
$$

where the (complex) function $Y$ is related to the Weyl tensor tetrad component by $Y=r \Psi_{4} ; \Delta$ is the horizon function $\Delta=r^{2}-2 M r$ and $\mathcal{T}$ is the source term determined by matter flows 10 . The polar metric perturbations correspond to the real part of $Y$, the axial ones to its imaginary part. At $r=3 M$ the term proportional to $Y_{, t}$ changes sign: depending on the relative sign between $Y_{, t t}$ and $Y_{, t}$, one may view $Y_{, t}$ either as a damping term (when the signs of both coefficients agree, i.e. for $r<3 M)$ or an antidamping term (otherwise). As argued by Krivan et al. [37], who analyzed in detail the general case of the Teukolsky equation for Kerr black holes, this is likely the origin of exponentially growing modes that appear when the equation is numerically solved by standard finite-differencing explicit methods. Although some attempts to delay in time the onset of the instability have been investigated in the literature [10, 37], it remains an open issue. As noted above, this effect is particularly disturbing in the presence of matter sources, since the instability is always occurring before that the late-time state of the system is reached.

On the other hand, using the tortoise coordinate, Eq. (19) reads

$$
Z_{, t t}-Z_{, r^{*} r^{*}}=V_{l} Z+S_{z}
$$

where the source term is the same one given by Eq. (21), with the derivatives with respect to $r$ consistently replaced by derivatives with respect to $r^{*}$. This equation does not present terms which may cause exponential growing modes, and, therefore, it permits stable evolutions.

\section{E. Initial data}

The nontrivial issue of how to specify suitable initial data (i.e. gravitational radiation free) in the presence of sources has been addressed to some extent in a number of works [10, 16, 18, 20, 38]. In particular, initial data suitable to describe point-like particles scattered by stars or falling onto black holes can be found in [18] and [38], respectively. The common procedure is to choose initial data such that the Hamiltonian and momentum constraints are satisfied at the initial temporal hypersurface. If the matter source is initially at rest, the initial conditions are time symmetric and the momentum constraint is automatically satisfied if the Hamiltonian constraint is. However, if velocity fields are present initially, the momentum constraint must be solved for too 18.

In the simulations reported in this work, we choose time-symmetric initial configurations where the matter distribution, shaped in the form of quadrupolar shells of dust or perfect fluid, is falling onto the central compact object from rest. Hence, we only need to consider the Hamiltonian constraint, Eqs. (11) and (15). This constraint is a single equation for three unknowns, $H, k$, and $\chi$. By setting $H=0$, one of the functions $k$ or $\chi$ can be specified freely and the constraint is then solved for the remaining one. Furthermore, the initial data are chosen so as to minimize the amount of gravitational radiation present initially. This is done by choosing $\chi=0$ and solving for $k$ with a given source $T_{00}$ 38]. Given $k$, the initial profile of $Z$ is then computed using Eq. (18). 
While this prescription should ensure that the initial data are free of any spurious GW content other than that due to the presence of the matter source, in practice this is not exactly the case, a certain amount of GWs being always present. Its origin is related to the finite value of the initial location of the shell. For such a configuration, the impossibility of specifying the GW contribution associated with the shell in a way consistent with its past history provokes a transient burst in which the excess GWs are radiated away. Similar situations were considered in Refs. 18, 38, 43], where the source of the perturbations was a particle orbiting around or scattered off a star or a black hole. In order to minimize this problem in our simulations, we freely evolve the perturbations without the hydrodynamics part until the initial unphysical burst of gravitational radiation leaves the numerical domain, taking the final profiles as the initial state for the actual simulations. In the black hole case, this simple procedure permits us to avoid completely any kind of initial data interference. For stars, however, this approach triggers the oscillations of the fluid modes. Hence, we further proceed by resetting $H=0$, in order to obtain the correct initial model.

\section{NUMERICAL FRAMEWORK}

The numerical schemes we have implemented to solve the hydrodynamics and perturbation equations (outlined in the preceding section) are used with some technical differences in both scenarios under study, neutron stars and black holes. The first difference is that, in the neutron star case, the overall grid is uniformly spaced in the radial coordinate $r$, while for the black hole it is uniformly spaced in the tortoise coordinate $r^{*}$. The numerical domain chosen to discretize the hydrodynamics equations is always smaller than that of the perturbation equations, which is extended in both directions, towards the horizon of the black hole (or the origin of coordinates in the case of the star) and towards large radii. This procedure avoids or minimizes the effect of the spurious reflection of waves at both boundaries. For stars, the hydrodynamics domain begins at the first cell outside the stellar surface, and extends up to $r_{\max }=108 \mathrm{~km}$. We choose the same resolution $(\Delta r \sim 0.07 \mathrm{~km})$ for both stellar models, so that the interior is covered with 146 points for model $\mathrm{A}$ and 200 points for model $\mathrm{B}$ which, we recall, is less compact. The hydrodynamics grid is covered with 1400 zones, a resolution chosen to ensure convergence. The external wave domain extends up to $r \sim 1500 \mathrm{~km}$ and is covered by roughly 22000 zones. On the other hand, in the case of black holes the hydrodynamics domain starts very close to the horizon so as to include as much as possible the peak of the potential barrier as well as its falloff toward $r^{*}=-\infty$. The sensitivity of our numerical results to the location of the inner boundary of the hydrodynamics domain is discussed in Sec. IV below.

Before turning to describe the numerical schemes im- plemented in the code, it is worth commenting that in a realistic scenario the mass of the compact object would grow in time as the accretion process proceeds. Such a possibility, however, has not been considered in the present simulations. Technically, this would require recomputing at each evolution time step the equilibrium structure of the star with a mass $M+\delta M(t)$ (or, analogously, increasing the black hole mass). In our simulations we simply assume that $\delta M \ll M$, neglecting the effect of a dynamically growing mass.

\section{A. Evolution of the external fluid}

The hydrodynamics part of our code is the same as that used in the simulations reported in Ref. [10]. In this code in order to evolve dynamically the infalling fluid shells, the general relativistic hydrodynamics equations are solved using a Godunov-type scheme (see e.g. 35] for details). In axisymmetry $\left(\partial_{\varphi}=0\right)$, the vector $\mathbf{U}$ of evolved quantities appearing in Eq. (7) is updated from time level $t^{n}$ to $t^{n+1}$ according to a conservative algorithm

$$
\begin{aligned}
\mathbf{U}_{i, j}^{n+1} & =\mathbf{U}_{i, j}^{n}-\frac{\Delta t}{\Delta r}\left[\hat{\mathbf{F}}_{i+1 / 2, j}^{r}-\hat{\mathbf{F}}_{i-1 / 2, j}^{r}\right] \\
& -\frac{\Delta t}{\Delta \vartheta}\left[\hat{\mathbf{F}}_{i+1 / 2, j}^{\vartheta}-\hat{\mathbf{F}}_{i-1 / 2, j}^{\vartheta}\right]+\Delta t \mathbf{S}_{i, j},
\end{aligned}
$$

where $\Delta t=t^{n+1}-t^{n}$, and $\Delta r$ and $\Delta \vartheta$ indicate the radial and angular grid spacing, respectively. In practice, a conservative, second-order, two-step Runge-Kutta algorithm is employed instead of Eq. (28). In the above equation $i$ and $j$ label the radial and angular zones, respectively. The numerical fluxes (e.g. $\hat{\mathbf{F}}_{i+1 / 2, j}^{r}$ ) are calculated at every cell interface using an approximate (linearized) Riemann solver built upon the characteristic information of the Jacobian matrices of the system. The reader is addressed to [35] for further details.

The matter model we choose for the accreting shells can be either dust or perfect fluid. In the former case, as $p=0$, the Riemann solver must be specialized accordingly to avoid divergences. For shells accreting onto black holes we employ dust, not only to allow for a direct comparison with the results of [10], but also because of numerical difficulties encountered when using a perfect fluid in regions very close to the event horizon. In Schwarzschild coordinates some metric components blow up at the horizon, which affects the evolution of hydrodynamical quantities. In particular, the coordinate flow velocity becomes ultra-relativistic and reaches the speed of light at the horizon, making the Lorentz factor infinite. If the inner boundary needs to be placed very close to the horizon to capture the fall off of the Zerilli potential (we have checked that $r^{*}=-50 M$ is a reasonable value; see below) the metric and hydrodynamical quantities, despite being regular, present steep radial gradients, which make the numerical evolution difficult. In our current code and with the maximum grid resolutions affordable, 
we have found severe limitations in the perfect fluid accretion case to move the innermost boundary past values of about $r^{*} \sim-3 M$ which, as we show below for the case of dust shells, are not yet close enough to the horizon to capture the gravitational wave emission unambiguously. We note that this numerical drawback can be entirely removed by using horizon-adapted coordinate systems such as ingoing Eddington-Finkelstein coordinates employed in simulations of perfect fluid accretion onto black holes in Ref. 39. Such a procedure for perfect fluid accretion may be attempted in future work.

However, for the case of neutron stars we do not encounter numerical difficulties in evolving perfect fluid shells. Therefore, in those simulations we do not consider quadrupolar dust shells, but only perfect fluid shells. This is also further motivated from the reflecting boundary conditions we impose at the surface of the star (see next paragraph), which may naturally lead to the appearance of shock waves which could not be treated in the case of dust.

The boundary conditions to impose upon the hydrodynamics variables completely depend on the case under study. In the black hole case, we adopt ingoing radial boundary conditions at the innermost grid point of the hydrodynamics domain, chosen as close as possible to the event horizon. As for the neutron star, the boundary conditions to impose to the infalling fluid shell as it arrives at the stellar surface should take into account the interaction between the shell and the star. As a result of the complexity in modeling this phenomenon within our perturbative approach, we impose reflecting boundary conditions at the inner edge of the hydrodynamics domain (i.e. the surface of the star), so that the star is seen by the external fluid as a hard sphere. This choice includes the most relevant effect; that is, the pressure gradient stops the infalling matter and avoids the violation of energy conservation that outgoing boundary conditions would introduce (as happens in the black hole case when the inner boundary is not close enough to the event horizon). Correspondingly, at the outermost radial boundary we impose stationary values of the so-called Michel solution at those grid points [40] at all times. We note that the stationary Michel solution is used in the entire hydrodynamics grid in order to provide a dynamically unimportant, spherically symmetric accreting atmosphere surrounding the fluid shell. In the angular direction, axial symmetry fixes the appropriate boundary conditions at the axis $(\vartheta=0$ and $\pi)$.

\section{B. Integration of the perturbation equations}

\section{Black hole case}

We can solve the Zerilli-Moncrief equation using either a standard three-level leapfrog method, at second-order in both space and time, or a second-order Lax-Wendroff scheme. Both methods have proved robust and stable enough for long time evolutions. In particular, they allow the computation of long-term features in the GW signal, namely the distinctive power-law tails following the black hole ringdown. However, when the leapfrog method is used, we find some high-frequency numerical noise of small amplitude at the very end of the tail. This numerical noise is not present when using the Lax-Wendroff scheme. Therefore, all results reported below for black hole spacetimes are obtained using this latter method. We note, however, that the second-order leapfrog produces noise-free results in the neutron star case. The particular form of such a scheme is discussed in the following section.

In order to apply the Lax-Wendroff method, the Zerilli-Moncrief equation is written as a first order hyperbolic system as follows:

$$
\partial_{t} \mathbf{U}+\partial_{r^{*}} \mathbf{F}=\mathbf{S}
$$

with

$$
\mathbf{U}=\left(\begin{array}{c}
Z \\
w
\end{array}\right), \quad \mathbf{F}=\left(\begin{array}{c}
Z \\
-w
\end{array}\right), \quad \mathbf{S}=\left(\begin{array}{c}
w \\
V_{l} Z+S_{z}
\end{array}\right),
$$

where we have introduced the variable $w=Z_{, t}+Z_{, r}$. The time update algorithm is given by

$$
\begin{aligned}
\mathbf{U}_{j}^{n+1} & =\mathbf{U}_{j}^{n}-\frac{\Delta t}{2 \Delta r^{*}}\left[\mathbf{F}_{j+1}^{n}-\mathbf{F}_{j-1}^{n}\right] \\
& +\frac{\Delta t^{2}}{2 \Delta r_{*}^{2}}\left[\mathbf{F}_{j+1}^{n}-2 \mathbf{F}_{j}^{n}+\mathbf{F}_{j-1}^{n}\right] \mathbb{A}+\Delta t \mathbf{S}_{j}^{n},
\end{aligned}
$$

where the matrix $\mathbb{A}$ reads

$$
\mathbb{A}=\left(\begin{array}{cc}
1 & 0 \\
0 & -1
\end{array}\right)
$$

To ensure stability of the code, the time step $\Delta t$ must satisfy the usual Courant-Fredrichs-Levy (CFL) condition, i.e. $\Delta t=\lambda_{\mathrm{CFL}} \Delta r^{*}$, with $\lambda_{\mathrm{CFL}}<1$.

The same radial resolution is used for the two domains (hydrodynamics and perturbations) present in the computational grid, namely $\Delta r^{*} \sim 0.04 M$. The angular domain of the hydrodynamics grid extends from 0 to $\pi$ and it is covered with 20 zones. As mentioned before, the hydrodynamics domain is within the wave domain, which is much larger. It extends from $r^{*}=-50 M$ to $r^{*}=30 M$ and is covered with about 2000 zones. The peak of the Zerilli potential is located at $r \sim 3.1 \mathrm{M}$ $\left(r^{*} \sim 1.9 M\right)$. Since the potential decays exponentially towards the horizon, $r^{*}=-50 M$ is a sufficiently small value to minimize the effects of the truncation on the GW signal (see below). On the other hand, in the positive $r^{*}$ direction it is not necessary to extend the hydrodynamics grid much beyond the position of the center of the shell, because the shell is always collapsing towards the black hole. Correspondingly, the grid for the perturbation equations extends from $r^{*} \sim-876 M$ to $r^{*} \sim 1250 M$. Standard Sommerfeld outgoing-wave 
conditions are imposed at the external boundary, and ingoing-wave conditions at the black hole horizon. Obtaining an optimal resolution in the simulations requires us to use $2 \times 10^{4}$ points from $r^{*}=0$ towards the horizon and some $3 \times 10^{4}$ zones in the opposite direction. Such a large number of zones, however, does not imply any numerical limitation in the code as solving the ZerilliMoncrief equation is a one-dimensional problem.

\section{Neutron star case}

The numerical algorithm used for solving the stellar perturbation equations does not use the gauge-invariant metric perturbation variable $\chi$, but instead $S=\chi / r$ (the equations are rewritten accordingly). The reason for this modification is that $\chi$ grows proportionally to $r$ for $r \rightarrow \infty$, while the amplitude of $S$ remains finite. This is an important property that helps to avoid unphysical instabilities of a numerical nature. The evolution equations for the independent variables $S$ and $H$ are discretized on a uniformly spaced grid and solved using the three-level leapfrog method. The remaining elliptic equation for $k$ is also discretized in space and then written as a tridiagonal linear system, which is solved at each time step inverting the corresponding matrix by a standard $L U$ decomposition.

In order to avoid numerical problems at the origin, the left interface of the first grid zone is chosen to coincide with the center of the star, $r=0$, while the surface of the star, $r=R$, is located at a cell center. The first point of the grid, $j=1$, is then located at $r=\Delta r / 2$, where $\Delta r$ is the radial grid spacing. Correspondingly, the surface, which is labeled by the $J$ cell, is located at $R=\Delta r / 2+(J-1) \Delta r$. As mentioned before, $S$ is evolved both inside and outside the star, while $H$ is only evolved inside the star according to Eq. (10) and at the surface with Eq. (12). The same grid spacing is chosen inside and outside the star.

The radial derivatives of $S, k$, and $H$ are discretized to second order,

$$
\begin{aligned}
{\left[A_{, r r}\right]_{j}^{n} } & =\left(A_{j+1}^{n}-2 A_{j}^{n}+A_{j-1}^{n}\right) / \Delta r^{2}, \\
{\left[A_{, r}\right]_{j}^{n} } & =\left(A_{j+1}^{n}-A_{j-1}^{n}\right) / 2 \Delta r
\end{aligned}
$$

(with $A \equiv S, k$ or $H$ ), but at $j=J$ we use backward second-order differencing for $H_{, r}$ as

$$
\left[H_{, r}\right]_{J}^{n}=\left(H_{J-2}-4 H_{J-1}+3 H_{J}\right) / 2 \Delta r .
$$

Similarly, the second time derivatives of $S$ (and $H$ ) are approximated as

$$
\left[S_{, t t}\right]_{j}^{n}=\left(S_{j}^{n+1}-2 S_{j}^{n}+S_{j}^{n-1}\right) / \Delta t^{2} .
$$

As for the black hole case, we impose standard Sommerfeld outgoing-wave boundary conditions at the outermost radial zone. Partial reflection from the external boundary is still present, but it can be minimized (or causally disconnect its influence) by placing the outermost point sufficiently far. At the origin of the radial coordinate $r=0$, all fields are regular and vanish [26]. For $l=2$, as the origin is chosen to lie at the interface of the first cell, there is no need for regularizing the fields, as had to be done in 16, 17.

In order to have an internal consistency test, we have studied the evolution of a generic enthalpy profile in the source-free case, computing the Zerilli-Moncrief function in two different ways. In the first option, it is computed algebraically at every temporal slice from $k$ and $\chi$ by Eq. (18), all over the external domain; in the second possibility, it is matched to $k$ and $\chi$ using Eq. (18) just at the stellar surface, and then it is evolved independently in the exterior region by solving Eq. (19). Both solutions agree very well, and only minor differences are observed during transient states.

\section{RESULTS}

\section{A. Black hole simulations}

As mentioned in the Introduction, time-dependent numerical simulations of the accretion of dust shells falling isotropically onto a Schwarzschild black hole were presented in [10]. The aim of those simulations was to characterize and estimate the gravitational radiation emitted in accretion events of finite-size objects and to compare the outcome with the point particle case amenable to semi-analytic investigations.

The time domain simulations discussed in [10] were based on the Newman-Penrose formalism, from which a master wave equation for black hole perturbations was derived by Teukolsky [11]. As mentioned above, in the case of a Schwarzschild black hole, the Teukolsky equation reduces to the $\mathrm{BP}$ equation. Here, we start by reexamining the GW emission of a Schwarzschild black hole as a result of the radial accretion of a quadrupolar dust shell. In this work, however, we use the inhomogeneous Zerilli-Moncrief equation for two different reasons: firstly, because it provides a direct comparison with the neutron star case and, at the same time, allows for crosschecking our results with those of [10]; secondly, the main motivation in reexamining this problem was the fact that the Zerilli-Moncrief equation allows for long and stable time evolutions. This property permits the investigation of long time features in the GW spectrum.

We consider an external matter distribution of total mass $\mu$, much smaller than that of the black hole, namely $\mu=0.01 M$. As in [10], the shell density distribution is parametrized according to

$$
\rho=\rho_{0}+\rho_{\max } e^{-\kappa\left(r-r_{0}\right)^{2}} \sin ^{2} \vartheta,
$$

where $r_{0}$ is the initial position of the center of the shell and $\kappa$ controls its width. The background density $\rho_{0}$ is chosen to be very small $\left(\sim 10^{-22} \mathrm{~km}^{-2}\right)$ to simulate the 

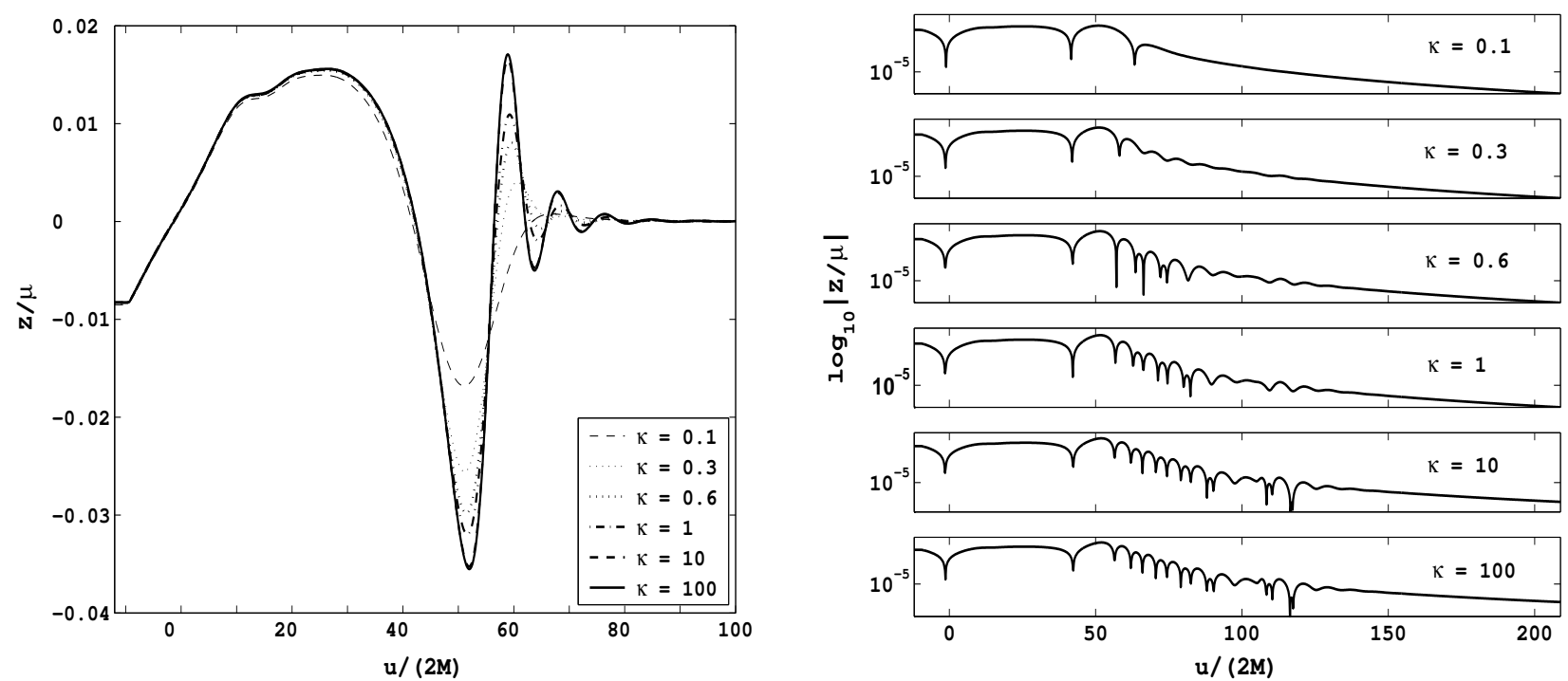

FIG. 1: Gravitational waves emitted by a Schwarzschild black hole excited by infalling quadrupolar shells of given compactness $\kappa$ [see Eq. [37)]. The right panels show the logarithm of the GW signals shown in the left panel in order to highlight the onset of the fundamental QNM ringing. The shells are falling from $r_{0}=15 M$ and the observer is located at $r_{\mathrm{obs}}=125 \mathrm{M}$ from the origin of coordinates.

vacuum outside the black hole. The angular structure of the matter density is assumed to have a quadrupolar profile, being given by $\sin ^{2} \vartheta$. The value of $\rho_{\max }$ is obtained from the condition that the volume integral of Eq. (37) equals $\mu$. Papadopoulos and Font [10] analyzed the dependence of the black hole QNMs excitation on the various parameters of the shell, namely its width, initial location, and its initial velocity field. Here, we begin by studying the excitation of the black hole QNMs varying the compactness of the shell, i.e. its width $\kappa$ and the position where it is at rest. We restrict ourselves first to shells which are falling from a fixed location $r_{0}=15 M$ and are initially at rest. The distant observer is located at $r_{\text {obs }}=125 \mathrm{M}$ from the origin of coordinates $\left(r^{*} \sim 133.5 M\right)$.

The response of the black hole as a result of the accretion process when shells of different widths $\kappa$ are considered is shown in the left panel of Fig. 1 in which we plot the time evolution of the Zerilli function. Note that in this figure we use retarded (observer) time $u=t-r^{*}$. The right panel shows the absolute values of the waveforms, but in logarithmic scale and separating the different models for clarity. The values of $\kappa$ are chosen in order to cover the range in which qualitative differences among the signals are observed. The process can be divided into three phases: (i) motion of the shell before the bulk reaches the peak of the Zerilli potential, from $u / 2 M \sim-10$ up to $u / 2 M \sim 50$, where the emission is purely due to the variation of the quadrupolar moment of the shell; (ii) shortly after $u / 2 M \sim 50$, when the bulk of the shell approaches the peak of the potential, $Z$ experiments a rapid variation due to the interaction with the potential barrier, resulting in a burst-like short sig- nal. For more compact shells, higher amplitudes of the Zerilli function are obtained. (iii) The last part of the signal, from $u / 2 M \gtrsim 55-60$ onward, is characterized by highly damped oscillations at early times (the fundamental QNM ringing of the black hole), followed by the power-law radiative tail at the end. In the right panel of Fig. 1 it is visible how the onset of the fundamental QNM ringing occurs only when $\kappa \geq 0.3$; i.e., wider (less compact) shells do not succeed in exciting the fundamental mode of the central black hole, but rather the signals show a large wavelength oscillation. This feature is in excellent agreement with the results reported by [10] using the BP equation. If $\kappa$ is too small, the impinging GWs cannot be fully transmitted beyond the potential barrier to cause the ringing of the fundamental QNM. Thus, the large wavelength oscillation is determined by the gravitational pulse driven by the shell, which is almost completely reflected back by the potential barrier. It is worth mentioning that, also in this case, the black hole spacetime reacts to the external perturbation, as we found that the other QNMs, characterized by frequencies lower than the fundamental one [41], can be slightly excited during the process. However, as a result of their high damping times, the energy that can be released through GWs is negligible with respect to that generated by the variation of the quadrupolar moment of the shell during the infall (roughly three orders of magnitude lower for $\kappa=0.1$ ). We note that the interaction of the shell with the black hole potential, even for small values of $\kappa$, is confirmed by the presence of the distinctive late-time decay.

It is known that the generation of the fundamental QNM ringing is associated with the peak of the potential [42]. The bulk of the shell crosses the peak at time 


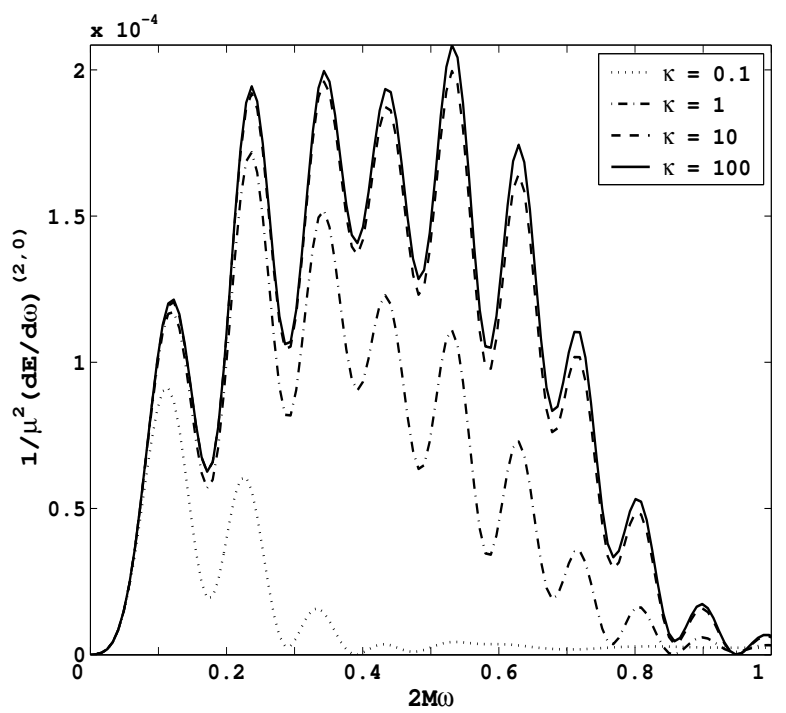

FIG. 2: Energy distribution for some selected values of $\kappa$ of the waveforms shown in Fig. 11 The power spectrum has a complex shape characterized by various distinctive peaks resulting from the interference between emitted and backscattered GWs (see text for details).

$t / 2 M \sim 50$, which is at retarded time $u / 2 M \sim 49$. As argued before, the maximum GW amplitude and the following QNM ringing are reached somewhere around this point. The end of the accretion process occurs at time $t / 2 M \sim 79.5$, when the center of the bulk $r_{0}$ reaches the innermost boundary of the hydrodynamical numerical domain. This time corresponds to a retarded time $u / 2 M \sim 104.5$.

The energy spectra for some selected values of $\kappa$ are shown in Fig. 2. For all values of $\kappa$, the spectrum displays a complex structure, with several distinctive peaks that are more or less evenly spaced. The spacing between the maxima is roughly given by 0.1 (in units of $2 M \omega$ ). The presence of these peaks is interpreted as an effect of the interference between the gravitational waves emitted by the shell during its motion and the radiation emitted at earlier times, which has already been backscattered by the potential. This interference fringes are not new. In fact, similar patterns were found by Lousto and Price [43] when studying the signal emitted by a point particle falling radially onto a Schwarzschild black hole from a finite distance $r_{0}$. However, some differences between their case and ours must be stressed. In Ref. [43], the initial data had some GWs content, and the authors argued that the evenly spaced bumps found in the energy spectra (whose amplitude and spacing depended on $r_{0}$ ) were mainly due to the interference between the initial data pulse and the GWs emitted by the infalling point particle during its motion. In Appendix $\mathbb{B}$ we confirm this result using a time domain code, by comparing the GWs energy spectra generated by a falling point particle with and without the initial GW content. In the latter case,

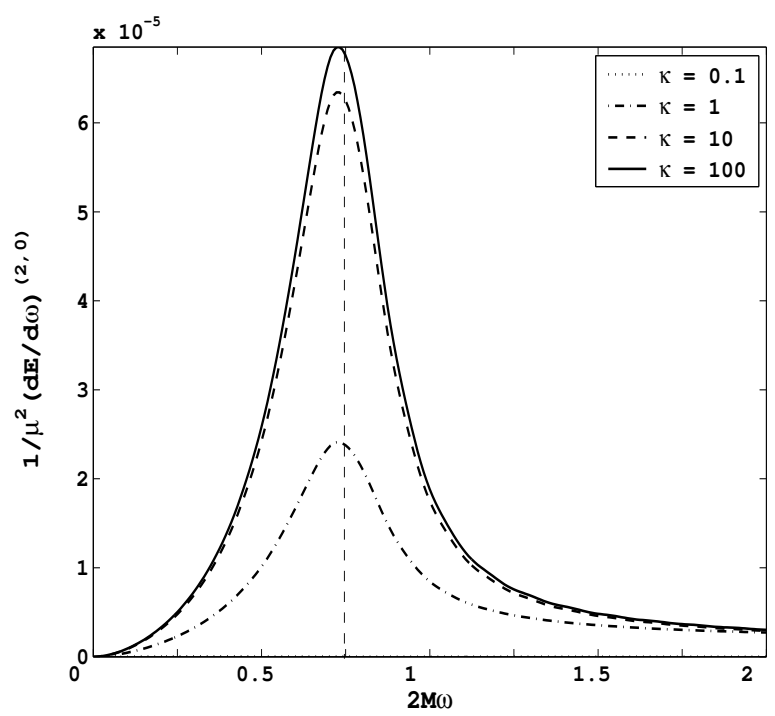

FIG. 3: Excitation of the $l=2$ fundamental QNM as a function of $\kappa$. The spectrum is computed selecting only the part of the signal corresponding to the ringing. The dashed vertical line indicates the fundamental QNM frequency $(2 M \omega \approx$ 0.7473) of a Schwarzschild black hole [41]. Note that for $\kappa=0.1$ the spectrum is barely visible at the bottom of the plot.

we find that the amplitude of the interference bumps is reduced. For the case of an imploding shell, despite the initial data effect being eliminated by construction, we find well-defined interference fringes in the energy spectra. The extended size of the matter distribution results in the existence of interference patterns even when the GW initial content is minimized.

As for the case of point particles [43], we notice that the part of the waveforms which strongly contributes to these features in the spectrum is that extending up to the second zero of the signal $(u / 2 M \sim 42)$, just before the burst. This portion of the waveform carries the imprint of the radiation emitted during the accretion process, before the bulk of the shell crosses the peak of the Zerilli potential. This is confirmed by the results shown in the spectra of Fig. 3] obtained considering only that part of the signal from the third zero of $Z$ onwards. This helps eliminate as much as possible the GWs contribution related to the motion of the shell and to dig out the actual QNM ringing signal. The spectrum of this late time signal closely corresponds to that of a Schwarzschild black hole radiating via the fundamental mode $(2 M \omega \approx 0.7473)$, whose frequency is marked by the vertical dashed line in the figure. The width of the peak is consistent with the damping time of the fundamental mode (see [41]), confirming that, if the black hole is successfully excited, the energy is radiated mostly in the fundamental mode.

In Fig. 4 we show the superposition of the QNM ringdowns for some selected values of $\kappa$. As expected, the power law of the late time tail does not depend on the 

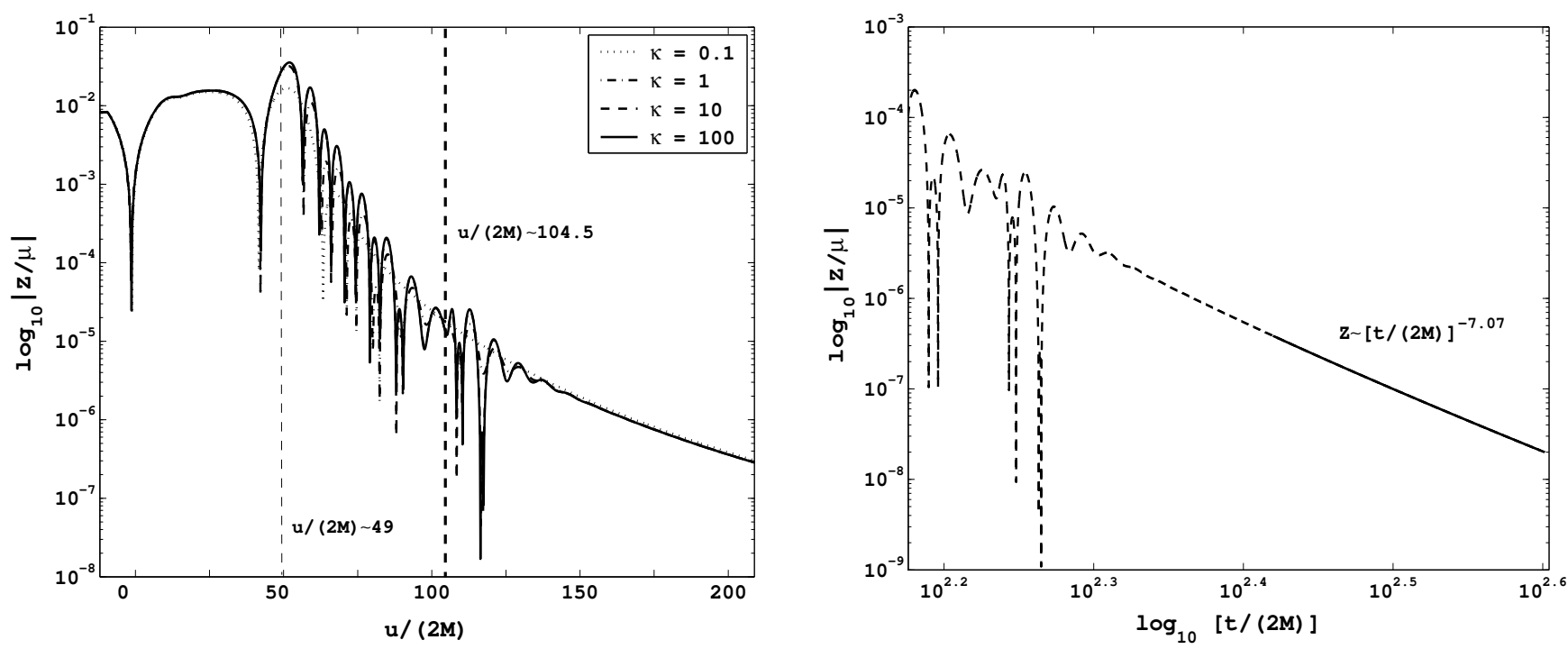

FIG. 4: Black hole QNMs ringdown and tails for various shell widths $\kappa$. The left panel shows that the GW burst resulting from the bulk of the shell crossing the peak of the Zerilli potential occurs at retarded time $u / 2 M \sim 49.5$ (first vertical dashed line). The late-time power-law tails are all perfectly superposed for the various values of $\kappa$ considered. At $u / 2 M \sim 104.5$ (second vertical dashed line), when the shell leaves the hydrodynamical numerical domain, a second ringing appears. This ringing is a numerical artifact (see text for details). The right panel depicts on a log-log plot the late time behavior of the longest simulation for a shell with $\kappa=10$. A fit to the solid line gives $Z \sim(t / 2 M)^{7.07}$, in excellent agreement with the analytic late-time falloff derived by Price [45], Eq. (38).

shell width $\kappa$, being all nicely overlapped. This important feature was not accessible to the simulations reported in [10], due to the appearance of numerical instabilities when solving the BP equation for sufficiently long evolution times. The late time behavior of gravitational perturbations was first studied in detail by Price [45] using analytic techniques. The most recent and exhaustive discussion of this topic can be found in [46]. The gravitational multipole perturbations with $l \geq 2$ are expected to fall off at large $t$ as

$$
Z \sim\left(\frac{t}{2 M}\right)^{-(2 l+3)}
$$

i.e. as $\sim t^{-7}$ in our case $(l=2, m=0)$. The best fit to the tails shown in the left panel of Fig. [4 which correspond to simulations that extend up to $\sim 3.67 \mathrm{~ms}$ of evolution $(u / 2 M \sim 209)$, gives $Z \sim(t / 2 M)^{-7.56}$. The somewhat large difference with the expected analytic value given by Eq. (38) is due to the fact that the signal has not reached yet the late-time state. In order to prove this, we perform a much longer run (up to $u / 2 M \sim 609$ ) employing a larger grid of $7 \times 10^{4}$ zones, which corresponds to roughly twice the number of zones used in the previous simulations. For this run, we set $\kappa=10$, keeping the same values for the remaining parameters. The right panel of Fig. [ 4 shows the results of this long run. The best fit to the late time signal is now $Z \sim(t / 2 M)^{-7.07}$, in close agreement with the expected value. In order to further improve the numerical results it simply suffices to perform even longer simulations employing larger grids.
Some more comments are relevant about Fig. 4 For $\kappa=1,10$, and 100 the signals present a strange feature, with a second ringing starting at $u / 2 M \sim 104.5$ (indicated by a thick vertical dashed line in the left plot). We have checked that this second ringing starts at the time when the center of the shell leaves the numerical domain through the innermost boundary. The appearance of this ringing seems to be a numerical artifact, as a small discontinuity in the fields is introduced when the center of the shell leaves the grid. The black hole reacts to this by emitting GWs in the form of the second ringing, until the late time tail is reached. This unphysical ringing could be avoided by extending the numerical domain as much as possible towards $r^{*}=-\infty$ (i.e. towards the event horizon). In practice, since the accretion processes we are interested in happen outside the horizon, where the peak of the Zerilli potential stands, it suffices to choose the innermost boundary of the hydrodynamics domain such that the exponential falloff of the potential is properly captured. The observation that most of the energy is released at low frequencies shows that the choice of the radial extent of the hydrodynamics domain with respect to the width of the Zerilli potential is of paramount importance to obtain the correct GW signals and the corresponding energy spectra. This effect is more important in the case of an extended shell, whose size changes with time due to the presence of tidal forces which tend to disrupt it before being swallowed by the black hole. It is the complex interaction with all the structure of the potential which determines the GW emission. 


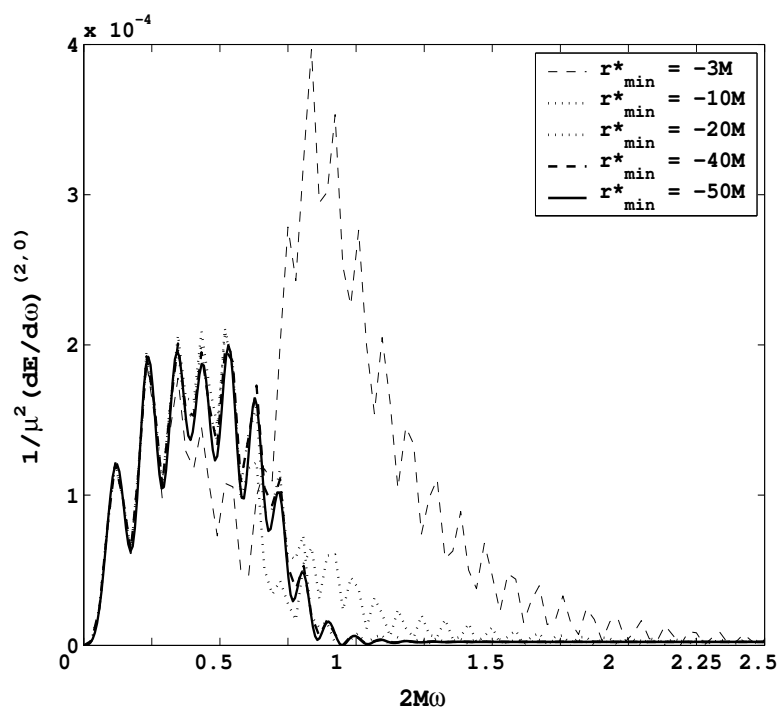

FIG. 5: Dependence of the GW energy spectra on the radial location of the innermost boundary $r_{\min }^{*}$ of the hydrodynamics numerical domain. The spectra plotted correspond to $\kappa=10$ quadrupolar shells falling from $r_{0}=15 M$, the GWs being extracted at $r_{\mathrm{obs}}=125 \mathrm{M}$. The unphysical high-frequency components disappear as $r_{\min }^{*}$ becomes smaller than about $-20 M$. The spectra converge for values of $r_{\min }^{*}$ as small as $-50 M$ (solid line).

In order to study how the radial extent of the hydrodynamical domain affects the waveforms and energy emission, we focus on an accreting shell of fixed width $\kappa=10$ and vary the radial location of the innermost boundary $r_{\min }^{*}$. The results of these simulations are shown in Fig. 5. We start with $r_{\min }^{*}=-3 M$ and gradually push $r_{\min }^{*}$ towards the event horizon, choosing the values $-10 M,-20 M,-40 M$, and $-50 M$. Although the low-frequency part of the spectra $(2 M \omega<0.4)$ is almost unaffected by the location of the inner boundary, high-frequency components become evident when $r_{\min }^{*}$ is larger than $-20 \mathrm{M}$. The energy spectra for $r_{\min }^{*}=-40 M$ and $r_{\text {min }}^{*}=-50 M$ are in practice identical. This means that such a radial extent suffices to capture all the relevant GW physics of the accretion process. However, using a less conservative value for the radial location of the inner boundary, e.g. $r_{\min }^{*}=-3 M$, results in an artificial spectrum which is roughly peaked again around the frequency of the fundamental mode. This was observed in [10] and attributed to QNM ringing. However, it is of completely numerical nature, with the same origin as the second ringing discussed in Fig. 4 when the shell crosses the inner boundary and leaves the grid, matter is artificially removed from the system, which violates energy conservation and produces the excitation of the black hole normal modes. If this happens at a relatively large radius such as $r^{*}=-3 M$, the effect is amplified. Moving the inner boundary closer to the event horizon shifts the second QNM ringing to later times, when the

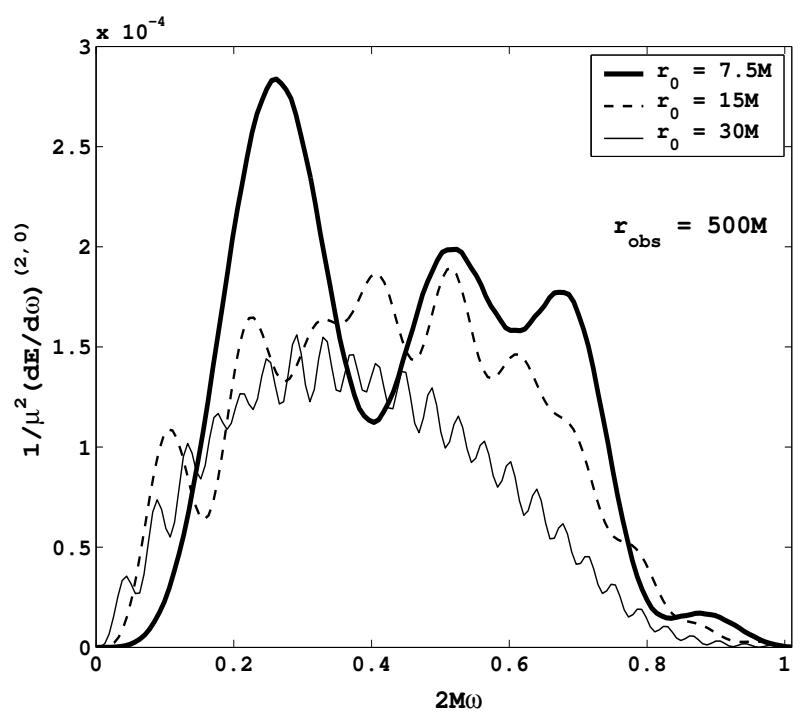

FIG. 6: Dependence of the energy spectra on the initial location of the shell $(\kappa=10), r_{0}=7.5 M$ (thick solid line), $r_{0}=15 M$ (dashed line), and $r_{0}=30 M$ (thin solid line). The number of interference fringes rapidly increases with the initial location of the shell.

signal is much weaker, and highly reduces the unphysical high-frequency part of the power spectrum. Therefore, the energy spectrum of the GW emission that one could expect in a realistic astrophysical scenario, during anisotropic accretion of matter onto a Schwarzschild black hole, is most likely to be a collection of interference fringes covering a wide range of low frequencies, than a single peak at the frequency of the fundamental mode of the black hole.

Next, we analyze the dependence of the energy spectra on the initial location of the shell. This is shown in Fig. [6 where we compare the spectra for three different initial locations, $r_{0}=7.5 \mathrm{M}, 15 \mathrm{M}$, and $30 \mathrm{M}$. The number of interference fringes rapidly increases with distance $r_{0}$. Furthermore, the correlation between the separation of the fringes and the initial position of the shell is evident: the larger the distance, the smaller the separation between consecutive maxima. In order to explain these modulations in the spectrum, we can follow the reasoning of Lousto and Price [43]. Given a GW signal $Z_{0}\left(t, r^{*}\right)$, its Fourier transform $\tilde{Z}_{0}\left(\omega, r^{*}\right)$ is defined according to Eq. (23). For large $r^{*}$, where the observer is located, the waveform represents only outgoing radiation, i.e. $Z_{0} \equiv Z_{0}\left(t-r^{*}\right)$, which gives

$$
\tilde{Z}_{0}(\omega, r)=\int_{-\infty}^{\infty} e^{-i \omega t} Z_{0}\left(t-r^{*}\right) d t=e^{-i \omega r^{*}} A(\omega),
$$

where

$$
A(\omega)=\int_{-\infty}^{\infty} e^{-i \omega u} Z_{0}(u) d u
$$

Let us now consider another GW signal $Z_{1}$ whose time 
delay with $Z_{0}$ is $T_{\text {shift }}$. This signal is $Z_{1} \equiv Z\left(r^{*}, t-T_{\text {shift }}\right)$, so that its Fourier transform reads

$$
\begin{aligned}
\tilde{Z}_{1}\left(\omega, r^{*}\right) & =\int_{-\infty}^{\infty} e^{-i \omega t} Z\left(t-T_{\text {shift }}, r^{*}\right) d t \\
& =e^{-i \omega T_{\text {shift }}} e^{-i \omega r^{*}} A(\omega) .
\end{aligned}
$$

Thus, the Fourier transform of the signal given by the superposition of $Z_{0}$ and $Z_{1}$ is given by

$$
\begin{aligned}
\tilde{Z}\left(\omega, r^{*}\right) & =\tilde{Z}_{0}\left(\omega, r^{*}\right)+\tilde{Z}_{1}\left(\omega, r^{*}\right) \\
& =e^{-i \omega r^{*}} A(\omega)\left(1+e^{-i \omega T_{\text {shift }}}\right) .
\end{aligned}
$$

Then, when computing the spectrum we have

$$
\omega^{2}\left|\tilde{Z}\left(\omega, r^{*}\right)\right|^{2}=4 \omega^{2}|A(\omega)|^{2} \cos ^{2}\left(\frac{1}{2} \omega T_{\text {shift }}\right) .
$$

Therefore, the modulation in the frequency spectrum is related to the characteristic time $T_{\text {shift }}$ which accounts for the delay between consecutive GW signals, $\delta \omega=2 \pi / T_{\text {shift }}$. From the measure of the peak spacing $\delta \omega$, we can thus infer the corresponding time shift. In the case of a radially infalling point particle, Lousto and Price [43] argued that this time shift roughly coincides with the infalling time. They use this empirical criterion as a rule of thumb to predict the variation of the spectra as $r_{0}$ is increased. We have found a similar correlation for our extended dust shells.

To close this section, we compute the total energy emitted in gravitational waves for shells accreting from the three initial locations considered previously, $r_{0}=7.5 \mathrm{M}$, $15 M$, and $30 M$. The energy emitted is computed by integrating in frequency the energy spectra of Fig. 6] where the integrals are calculated using a standard trapezoidal rule. As we do in the point-particle case (see Appendix [B] , it is convenient to use as a reference quantity the ratio $2 M / \mu^{2} E^{20}$. Table IIIsts the values of the energy for the three positions $r_{0}$ considered. We note that the third value (for $r_{0}=30 M$ ) may be slightly underestimated due to some inaccuracies in the resolution of the power spectrum. It is worth stressing that, irrespective of the location $r_{0}$ of the shell, the values reported in Table II are smaller by roughly two orders of magnitude than those obtained in the point-particle limit 38, 43. [Lousto and Price [4] give $\left(2 M / \mu^{2}\right) E^{20}=1.64 \times 10^{-2}$ for $r_{0}=30 M$ and $1.43 \times 10^{-2}$ for $\left.r_{0}=10 M\right]$. The reduction we find in the total energy emitted in gravitational waves is a consequence of the finite size of the shells considered in the present work.

We note that in the numerical simulations reported in 10] the estimation of the energy yielded considerably larger values than the ones reported here, asymptoting towards a third of the point-particle limit 9$]$ as the compactness of the shell was increased. We argue that such a high value is overestimated, because it was affected by errors induced by the location of the innermost boundary of the hydrodynamics grid $\left(r^{*}=-3 M\right)$, as we have shown in Fig. [5 An indirect validation of the current
TABLE II: Total energy emitted in gravitational waves for shells accreting onto a Schwarzschild black hole from different distances $r_{0}$.

\begin{tabular}{cc}
\hline \hline$r_{0} / M$ & $\left(2 M / \mu^{2}\right) E^{20}$ \\
\hline 7.5 & $1.09 \times 10^{-4}$ \\
15 & $9.49 \times 10^{-5}$ \\
30 & $7.20 \times 10^{-5}$ \\
\hline
\end{tabular}

estimation of energy emission comes from an inspection of the findings of Shapiro and Wasserman [5]. These authors compute the total energy radiated in gravitational waves (while here we restrict to the $l=2$ multipole) from non-spherical dust clouds falling into a black hole from infinity. For any of the models considered, they find that the energy released in GWs is always smaller by at least two orders of magnitude with respect to the point-particle limit, with thinner clouds more efficient than wider ones.

\section{B. Neutron star simulations}

We turn now to consider the case of quadrupolar perfect fluid shells accreting onto neutron stars. Two neutron star models are considered, models A and B, whose characteristics (mass and radius) have been described in Sec. IIA above. Initially, the quadrupolar shell is surrounded by a background fluid (an "atmosphere") satisfying the stationary and spherically symmetric Michel solution [40]. The initial rest mass density profile is given by Eq. (37), where $\rho_{0}$ is now the profile consistent with the Michel solution. As in the black hole case, the mass of the shell is $\mu=0.01 M$, which corresponds to a maximum density of $\rho_{\max } \sim 3.5 \times 10^{-6} \mathrm{~km}^{-2}$ when we fix its width to $\kappa=1$. The (inhomogeneous) density profile of the atmosphere $\rho_{0}$ is roughly three orders of magnitude lower than $\rho_{\max }$. The shell obeys a polytropic $\left(p=\mathcal{K} \rho^{\gamma}\right)$ EOS with $\mathcal{K}=0.01 \mathrm{~km}^{2 / 3}$ and $\gamma=4 / 3$. The initial internal energy profile is obtained from $\rho$ and $p$ through the first law of thermodynamics as $e=p /[(\gamma-1) \rho]$. The shell is initially at rest at $r_{0}=20 \mathrm{~km}$, and the GW signal is extracted at $r_{\mathrm{obs}}=250 \mathrm{~km}$.

We first present an overview of a typical evolution for both models, in order to have an immediate insight on the dependence of the gravitational wave signal on the compactness of the star. As a result of the reflecting boundary conditions imposed at the surface of the star and the existence of an extended atmosphere, the accretion process is now followed by the formation of shock waves which propagate off the stellar surface. As in the case of the black hole, the impact of the shell perturbs the star and triggers its quasinormal modes of pulsation.

Figure 7 displays the time evolution of the metric functions $\chi$ and $k$, as well as the enthalpy perturbation $H$, for both models. Model A (B) is presented in the left (right) 

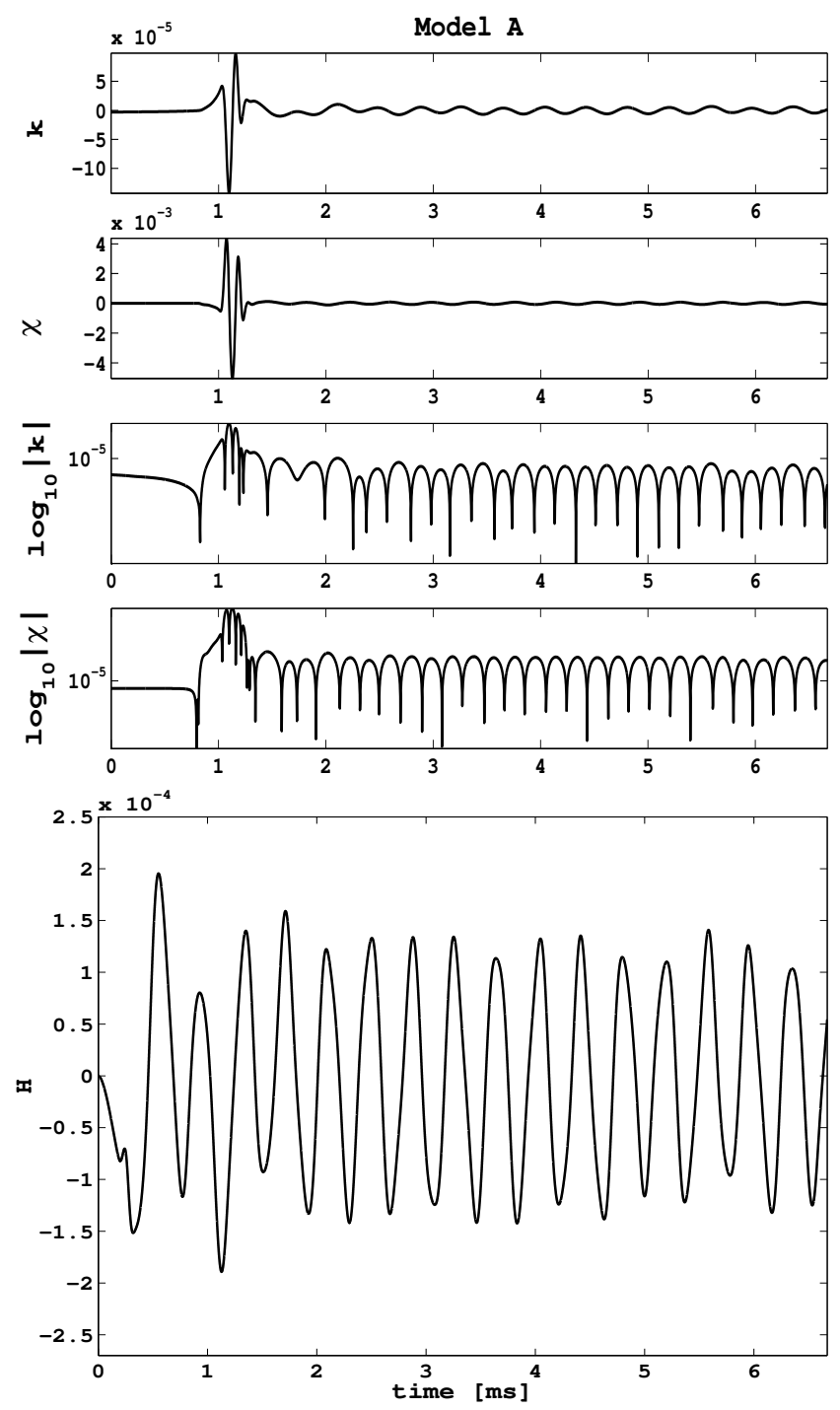
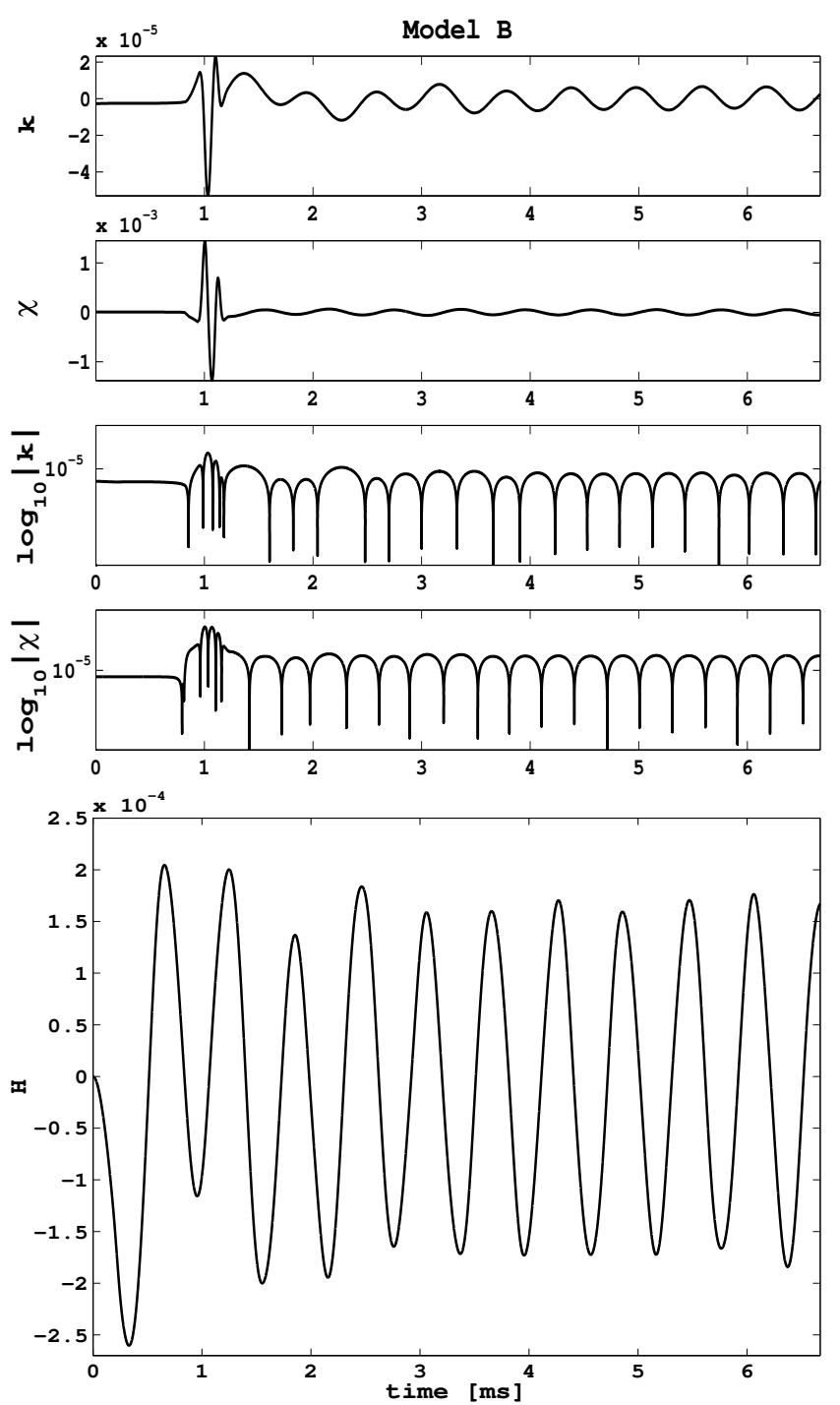

FIG. 7: Time evolution of the metric variables $k$ and $\chi$ and of the enthalpy perturbation $H$ for the two neutron star models considered. The left panels correspond to model A and the right panels to model B. The burst of gravitational radiation and the subsequent metric and fluid oscillations are clearly identified for both stellar models. The infalling perfect fluid shell has an initial compactness $\kappa=1$ and is located at a distance $r_{0}=20 \mathrm{~km}$. Note that, contrary to the previous figures, the time is now given in ms.

panels. In this figure we show the all the variables and the logarithms of the absolute values of the metric functions, in order to highlight the oscillating modes of the two stars. Correspondingly, Fig. [8 exhibits the time evolution of the Zerilli-Moncrief function for both models, in linear and logarithmic scales, computed at every time step according to Eq. (18). In the behavior of $Z$, as well as in the time evolution of $\chi$ and $k$ shown in Fig. 7 the three phases discussed in the preceding section for the black hole case are also visible: first, the infalling phase, when the bulk of the shell is evolving outside the star, gradually approaching it, which is characterized by a steady increase of the amplitude of the signal. This phase is very short as the shell is initially located very close to the stellar surface. Second, a burst-like peak appears in the GW signal, which, as found in relativistic simulations of gravitational core collapse [47], coincides with the moment when the shell reaches the surface, creating a shock wave which propagates off the surface. Finally, there is the ringdown phase, characterized by a GW signal which is not exactly monochromatic as a result of the complex interaction between the gravitational field of the star and the layers of fluid captured on top of the stellar surface in the process of readjusting themselves to a new stationary solution. The duration of the ringdown phase is now much longer than for the Schwarzschild black hole case discussed previously, as the damping time of the fundamental mode of the fluid is considerably larger. We note 

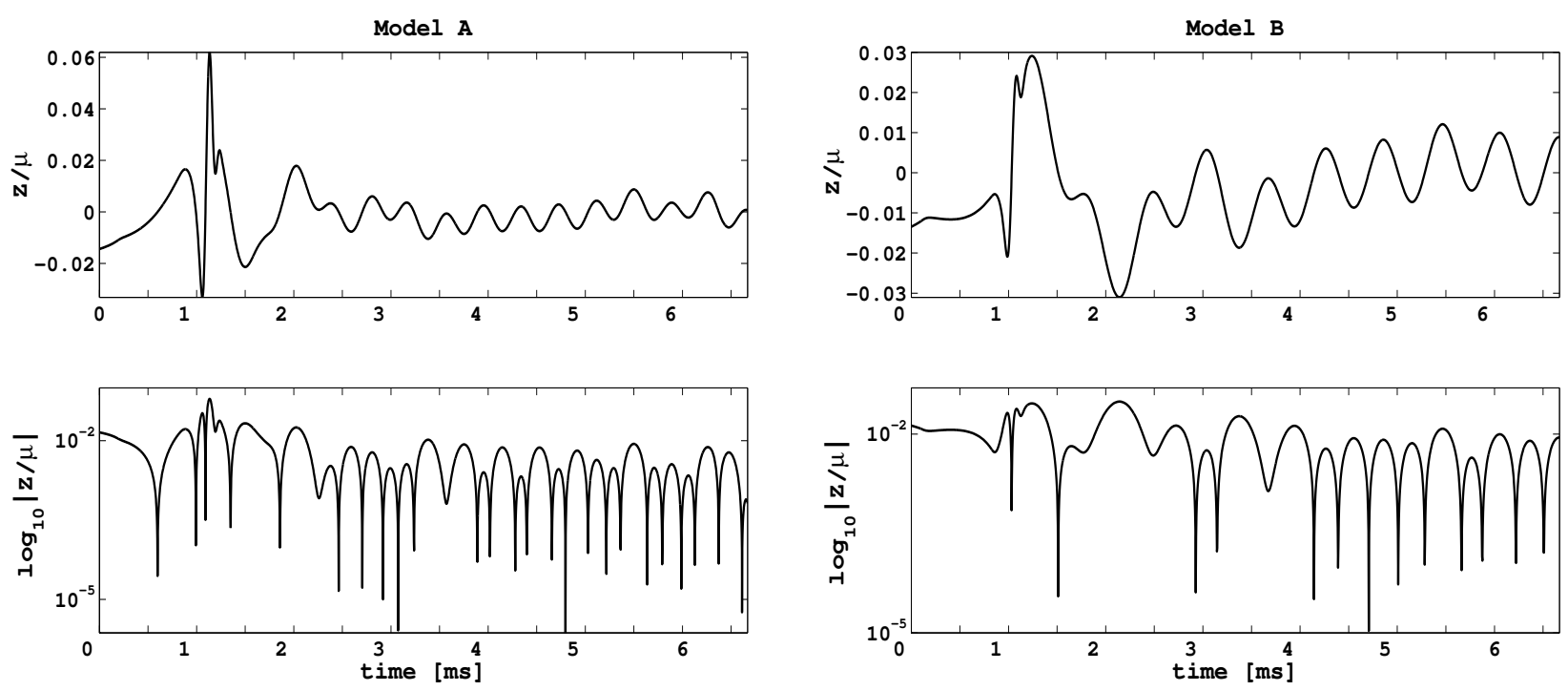

FIG. 8: Time evolution of the Zerilli-Moncrief function for the two stellar models considered. In close qualitative agreement with the results from gravitational core collapse simulations [47], the burst in the Zerilli-Moncrief function is associated with the moment of the bounce of the accreting shell at the stellar surface, and occurs at a retarded time of about $1 \mathrm{~ms}$.

that despite the waveforms obtained in our simulations showing a remarkable resemblance with those obtained in core collapse simulations [47], there are also important differences in the post-bounce phase dynamics, as the ringdown of the neutron star lasts for much longer times. In our idealized setup, with a perfect fluid, these pulsations are not quickly damped by the existence of a dense envelope surrounding the star, as happens in the core collapse situation.

The bulk of the accreting matter (the center of the shell) reaches the stellar surface at $t_{A} \sim 0.2 \mathrm{~ms}$ for model $\mathrm{A}$ and $t_{B} \sim 0.14 \mathrm{~ms}$ for model $\mathrm{B}$. At $r_{\mathrm{obs}}$, where the observer is located, the GW signals generated by these events are delayed in time by

$$
\Delta t=\left[r_{\mathrm{obs}}-R+2 M \log \left(\frac{r_{\mathrm{obs}}-2 M}{R-2 M}\right)\right] .
$$

Therefore, the signal generated by the matter bouncing back at the stellar surface reaches the observer at times $t_{A}^{\text {obs }}=t_{A}+\Delta t_{A} \approx 1.06 \mathrm{~ms}$ and $t_{B}^{\text {obs }}=t_{B}+\Delta t_{B} \approx 0.97 \mathrm{~ms}$. These values are consistent with the results of Fig. 17 where the waveforms of $k$ and $\chi$ are found to show bursts of large amplitude followed by highly damped oscillations sometime around these values. This observation is particularly confirmed in the logarithmic plots of the GW signals. After the short-lived ringing phase lasting for a fraction of half a millisecond after the burst, only the fundamental oscillation mode of the star is visible in all variables plotted in Figs. 7 and 8

The qualitative behavior found in the GW emission is the same for both models. Only quantitative differences appear in the amplitudes at the maximum, which are systematically larger by roughly a factor of 2 for the more compact model (A). This difference in ampli- tude becomes more apparent in the energy spectra of the Zerilli-Moncrief function shown in Fig. 9] The solid lines in this figure are obtained by Fourier transforming the complete signal, i.e. also including the contributions from the shell infall phase which precedes the burst. Correspondingly, the dashed lines are obtained from truncated waveforms, in which we only take into account the contribution from the beginning of the burst $(\sim 0.9 \mathrm{~ms})$ onward. We notice that, despite the short evolution times of our simulations, the $f$ mode frequency is very well identified in the spectra, the relative difference with respect to the values listed in Table I being roughly of $1 \%$. The value of the $f$ mode frequency is indicated with a circle in Fig. 9] Furthermore, it is worth stressing that we obtain the same qualitative spectra as for the black hole case - a complex pattern with interference fringes with the addition, in the neutron star case, of a high peak standing at the frequency of the $f$ mode. As we discussed in the preceding section for a Schwarzschild black hole, the comparison between the spectra obtained from the total and the truncated Zerilli signal shows that the interference fringes are produced by the interaction of the infalling fluid and the star.

There are other small differences between the two stellar models. The more compact model (A) is more efficient at high frequencies than model $\mathrm{B}$. This is directly correlated with the large amplitudes attained by the peaks of the Zerilli-Moncrief function in the time domain (cf. Fig. 8). The broadband spectrum of model A presents one broad peak with a maximum at about 7 $\mathrm{kHz}$, which is however too low to be identified with the first $w$ mode (see Table 【). As mentioned before, its origin should be related to the motion of the fluid shell and its interference with the gravitational field of the star, 

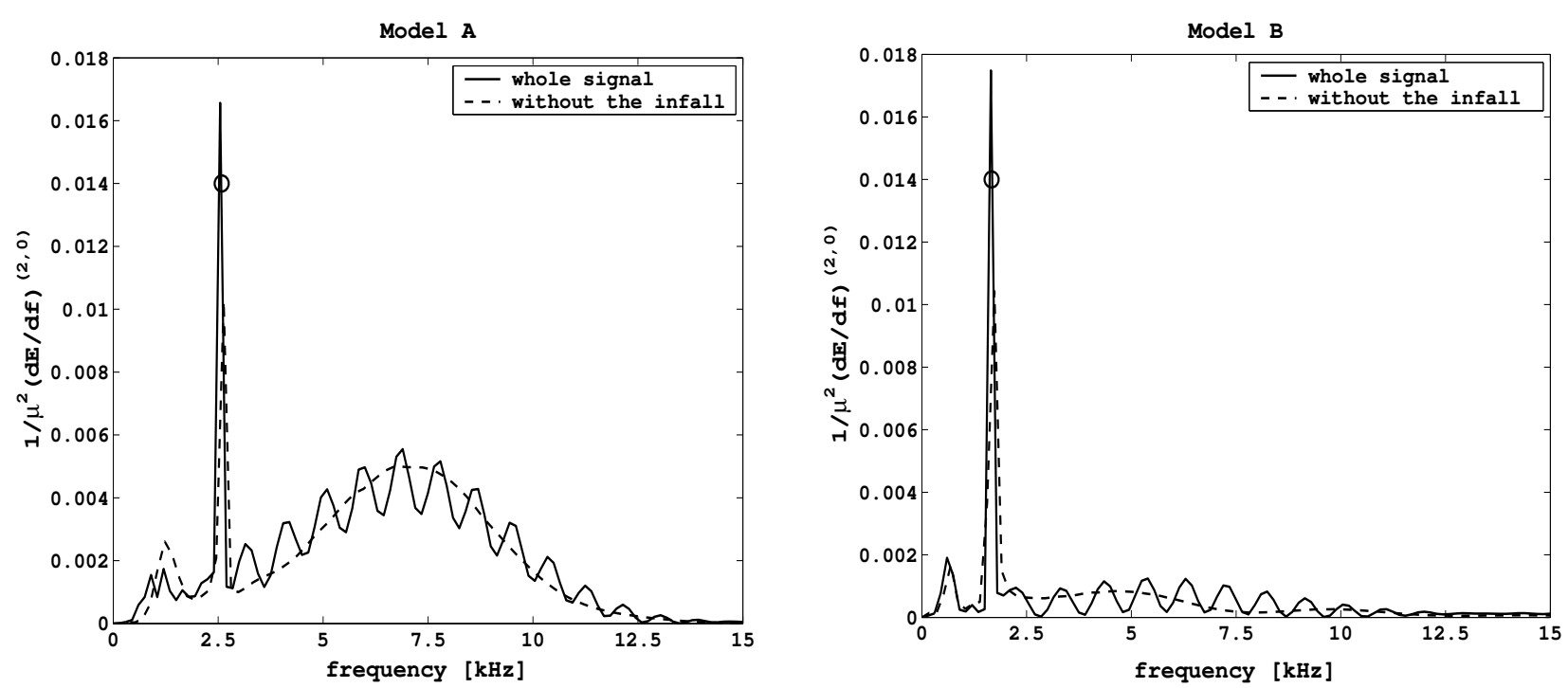

FIG. 9: Energy spectra corresponding to the time evolution of the Zerilli-Moncrief function depicted in Fig. 8 Model A (B) is shown on the left (right) panel. The solid lines show the spectra for the entire signal while the dashed lines are obtained removing that part of the waveform which corresponds to the infall phase of the shell. The spectra of the entire signal show characteristic interference fringes, qualitatively similar to those found in the black hole case, with a large amplitude peak standing at the frequency of the $f$ mode. The value of the frequency of the $f$ mode from Table $\prod$ is indicated by a circle. The spectra of the truncated signals show no evidence of the interference patterns.

or, in other words, on the reflection of the GWs pulse associated with the shell distribution with the "external" Zerilli potential. Thus, this broad feature depends on details of the accretion dynamics rather than on the intrinsic characteristics of the star.

We note that in both spectra there appears a small amplitude peak at frequencies lower than that of the $f$ mode for each model. This second peak is associated with oscillations of that part of the external fluid that has been gravitationally captured by the central neutron star as a result of accretion. In order to illustrate this affirmation, we plot in Fig. 10 the energy spectra corresponding to two fluid shells which only differ on the mass $(\mu=0.01 M$ and $0.001 M)$, falling onto stellar model B from the same distance, $r_{0}=20 \mathrm{~km}$. The $f$ mode is also properly excited for the less massive shell. Furthermore, the interference fringes in the high frequency part of the spectra coincide to high precision for the two shells considered, after normalizing to the corresponding shell masses. However, the structure of the low frequency peak is the only feature of the spectra which is influenced by the mass of the shell. We argue that the existence of this unphysical low frequency peak is an artifact produced by the boundary conditions. Notice, once more time, that we model the surface of the star as a hard surface, with reflecting boundary conditions. In a realistic scenario, the accreted matter would not simply bounce at the stellar surface, but it would rather interact with the neutron star envelope, resulting in heating and suffering nuclear reactions, until it is reabsorbed by the star.

As we did for the black hole case, let us now close this section by computing the total energy emitted in gravitational waves for the two stellar models considered. For doing so we integrate in frequency the spectra appearing in Fig. 9 The result of the integration yields

$$
\begin{aligned}
& E_{A}^{20} \simeq 3.02 \times 10^{-8} M_{\odot} c^{2}, \\
& E_{B}^{20} \simeq 8.36 \times 10^{-9} M_{\odot} c^{2} .
\end{aligned}
$$

Model A is hence slightly more efficient than model B concerning GW emission. We note in passing that these values are as small as those found in core collapse simulations [47], although such a comparison only makes sense in qualitative terms. Finally, the mass of the shell radiated in gravitational waves is

$$
\begin{aligned}
& E_{A}^{20} \simeq 2.15 \times 10^{-6} \mu, \\
& E_{B}^{20} \simeq 5.97 \times 10^{-7} \mu .
\end{aligned}
$$

\section{CONCLUSIONS}

In this paper we have presented a detailed analysis of the gravitational radiation induced by the anisotropic accretion of quadrupolar (dust or perfect fluid) shells onto non-rotating black holes and neutron stars. The numerical framework for our simulations is based upon a hybrid procedure in which the linearized equations describing metric and fluid perturbations are coupled to a fully nonlinear hydrodynamics code that calculates the motion of the accreting matter. These equations are 


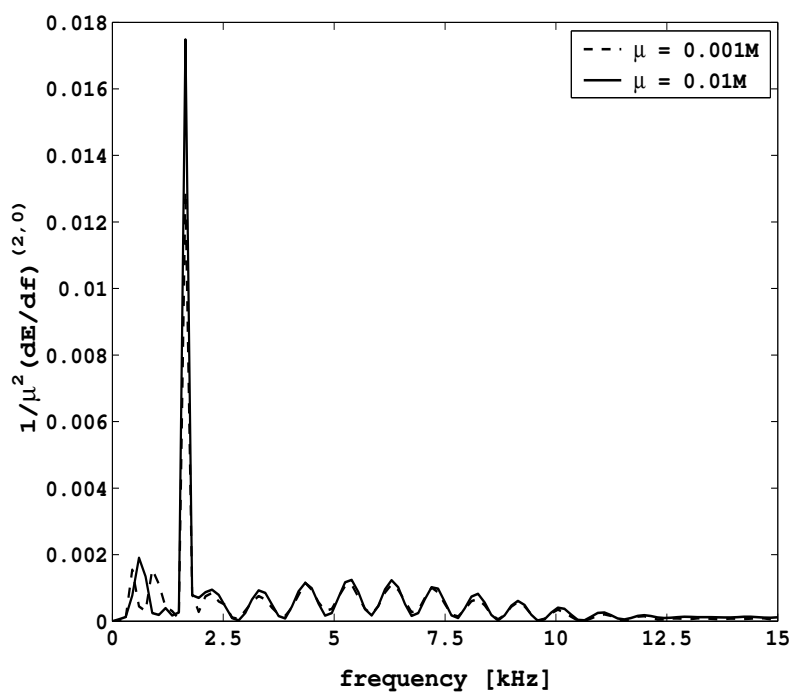

FIG. 10: Energy spectra for two fluid shells differing on the initial mass $\mu$ and infalling from a distance of $r_{0}=20 \mathrm{~km}$ onto stellar model B. The solid line corresponds to $\mu=0.01 M$ and the dashed line to $\mu=0.001 M$. The $f$ mode excitation and the high frequency part of the spectra coincide to high precision for the two shells considered. Only the low frequency peak is affected by the different mass of the shell.

integrated numerically in axisymmetry using advanced computational techniques. The numerical schemes developed have proved to be stable and highly accurate. Regarding the perturbation equations, the two main technical changes with respect to previous works reported in the literature are the use of the Zerilli-Moncrief function and the in-built conservation of the Hamiltonian constraint. The hydrodynamics equations are solved using high-resolution shock-capturing schemes based upon approximate Riemann solvers. We have shown that a perturbative approach can be used as a very effective tool to understand the basic gravitational physics operating in interesting astrophysical situations, extending the information which can be gained from the study of point-like particles infalling onto black holes or orbiting around them. In this context, our hybrid approach can be extremely useful to understand the gravitational radiation from astrophysical systems, complementing the whole machinery of full numerical relativity.

The simple fluid configurations analyzed in this paper ascertain, however, that the effects of the extended structure of the accreting matter are indeed highly relevant for gravitational wave emission. In the black hole case, most of the energy is released at frequencies lower than that of the fundamental QNM of the black hole, the spectrum consisting of a complex pattern, mostly produced during the accretion process rather than in the ringdown phase. Therefore, the GW emission that could be expected from a realistic astrophysical scenario in which accretion operates (e.g. after gravitational collapse of a massive star) is more likely to be a sort of interference fringe, cover- ing low frequencies, than a single monochromatic peak at the frequency of the fundamental mode of the black hole. This result, which went unnoticed in earlier hydrodynamical simulations by [10], was already observed by Lousto and Price [43] in the case of a point-like particle falling onto a black hole (see Appendix [B]), a situation amenable to semi-analytic investigation. However, we have shown that the appearance of interference fringes is very much amplified when the accreting fluid is an extended shell of finite size which, in turn, reduces the amount of energy which is released in gravitational waves to some two orders of magnitude below the point-particle value. It is interesting to notice that ground-based interferometric detectors attain the maximum sensitivity at frequencies considerably lower than the QNMs of stellar mass black holes. For this reason they are usually not considered as optimal sources for detection. However, to the light of our findings, the process of accretion appears to be quite more effective at frequencies about a factor of 2-3 lower than that of the black hole fundamental mode, and therefore the chances of detecting gravitational wave signals from such scenarios may be larger than expected. It must be stressed that the interference pattern does not happen only because of the GW content of the initial data, but it is a distinctive feature due to the extended size of the object, resulting from the interaction between the infalling matter and the backscattered waves.

In the neutron star case the qualitative results are similar, but a considerable part of the energy is emitted at the frequency of the fundamental mode. We have shown that the $f$-mode of the star is the only one excited at significant levels, and that the high frequency spectrum is quite sensitive to the spatial distribution of the accreting matter, making the contribution of the spacetime $w$-modes of the star difficult to be identified. The waveforms obtained show a remarkable resemblance with those obtained in core collapse simulations [47], despite the fact that we are considering a very different scenario. The main difference is that in our case the ringdown of the neutron star lasts for much longer times: the pulsations are not quickly damped by the existence of a dense envelope surrounding the star, as happens in the core collapse situation.

The results reported in this paper can partly be considered as a necessary assessment of our numerical approach in anticipation of the study of more interesting astrophysical scenarios, namely the excitation of QNMs from perfect fluid thick accretion tori orbiting around compact objects (see e.g. [48, 49] and references therein), which will be presented elsewhere 32].

\section{ACKNOWLEDGMENTS}

We thank L. Villain, E. Berti, V. Ferrari, R. De Pietri, and E. Onofri for discussions, suggestions and assistance, and K. D. Kokkotas and L. Rezzolla for a critical reading of the manuscript. This work has been sup- 
ported by the EU Programme "Improving the Human Research Potential and the Socio-Economic Knowledge Base" (Research Training Network Contract HPRN-CT2000-00137) and the Spanish MCyT grant AYA 20013490-C02-01. J.A.P. is supported by a Ramón y Cajal contract from the Spanish MCyT. All the computations were performed on the INFN Albert100 cluster for numerical relativity of the University of Parma.

\section{APPENDIX A: GENERAL SOURCE TERM FOR THE ZERILLI-MONCRIEF EQUATION}

In this appendix we derive the source term given in Eq. (21) for a general stress-energy tensor. We use the normalization of the Zerilli-Moncrief function $\phi$ given in Ref. 27], although the function we evolve in the numerical simulations is rescaled as $Z=2 \phi / \lambda$. The inhomogeneous equation written using the frame derivatives notation of [27] reads

$$
-\ddot{\phi}+\phi^{\prime \prime}+\nu \phi^{\prime}-V_{\phi} \phi=S_{\phi},
$$

where $V_{\phi}$ is the potential and $S_{\phi}$ the source term. As shown by Moncrief [31], $\phi$ can be written in terms of $k$ and $\chi$ as

$$
\phi=A(r) \chi+B(r) k+C(r) k^{\prime}
$$

where

$$
\begin{aligned}
A & =\frac{2 r^{2} e^{-2 b}}{(\lambda-2) r+6 M}, \\
B & =\frac{r(r \lambda+2 M)}{(\lambda-2) r+6 M}, \\
C & =-r A e^{b} .
\end{aligned}
$$

The derivation of the source term $S_{\phi}$ follows from the knowledge of the source terms in the evolution equations for $\chi$ and $k$ that can be written in vacuum [27]. Using Eq. A2 in Eq. A1 we get

$$
\begin{aligned}
-\ddot{\phi}+\phi^{\prime \prime}+\nu \phi^{\prime} & =A\left(-\ddot{\chi}+\chi^{\prime \prime}\right)-B \ddot{k} \\
& +\left[B+2 C^{\prime}+\nu C\right] k^{\prime \prime} \\
& +C\left[-\left(k^{\prime}\right)^{\cdot}+k^{\prime \prime \prime}\right] \\
& +L\left(k, \chi, k^{\prime}, \chi^{\prime}\right),
\end{aligned}
$$

where $L$ is a linear operator acting on $k, \chi, k^{\prime}$ and $\chi^{\prime}$, whose explicit form is not relevant for the computation of the source. In fact, in Ref. [27] it is shown that all terms which are linear in the fields $\chi$ and $k$ and their first-order spatial frame derivatives merge together to build the Zerilli potential. The frame derivatives do not commute [26],

$$
(\dot{k})^{\prime}-\left(k^{\prime}\right)^{\cdot}=-\nu \dot{f},
$$

so that

$$
\left(k^{\prime}\right)^{*}=(\ddot{k})^{\prime}+2 \nu \ddot{k},
$$

$\dot{\nu}=0$ being on a static background. Equation A6 becomes then

$$
\begin{aligned}
-\ddot{\phi}+\phi^{\prime \prime}+\nu \phi^{\prime} & =A\left(-\ddot{\chi}+\chi^{\prime \prime}\right)+B\left(-\ddot{k}+k^{\prime \prime}\right) \\
& +\left(\nu C+2 C^{\prime}\right) k^{\prime \prime}-2 \nu C \ddot{k} \\
& +C\left(-\ddot{k}+k^{\prime \prime}\right)^{\prime}+L\left(k, \chi, k^{\prime}, \chi^{\prime}\right) .
\end{aligned}
$$

Next, we can write the evolution equations for $k$ and $\chi$ in a Schwarzschild spacetime and the Hamiltonian constraint as

$$
\begin{aligned}
-\ddot{\chi}+\chi^{\prime \prime} & =L\left\{k, \chi, \chi^{\prime}\right\}+T_{\chi} \\
-\ddot{k}+k^{\prime \prime} & =L\{\chi, k\}-(2 W+\nu) k^{\prime}+T_{k} \\
k^{\prime \prime} & =L\left\{\chi, k, \chi^{\prime}, k^{\prime}\right\}+T_{\mathcal{H}},
\end{aligned}
$$

with $T_{\chi}, T_{k}$ and $T_{\mathcal{H}}$ being the sources induced by the matter distribution. From these equations one obtains

$$
\left(-\ddot{k}+k^{\prime \prime}\right)^{\prime}=L\left\{k, \chi, k^{\prime}, \chi^{\prime}\right\}-(2 W+\nu) T_{\mathcal{H}}+T_{k}^{\prime},
$$

so that the source of the Zerilli equation is found to be

$$
\begin{aligned}
S_{\phi} & =A T_{\chi}+(B+2 \nu C) T_{k} \\
& +\left[2 C^{\prime}-2(W+\nu) C\right] T_{\mathcal{H}}+C T_{k}^{\prime} .
\end{aligned}
$$

In polar radial coordinates the sources are, explicitly,

$$
\begin{aligned}
T_{\mathcal{H}}=-8 \pi S_{\mathcal{H}}, \\
T_{\chi}=-16 \pi e^{2 a} S_{\chi}, \\
T_{k}=8 \pi\left\{-e^{-2 a} T_{00}^{\ell m}+e^{-2 b} T_{11}^{\ell m}\right. \\
+4 \frac{e^{-2 b}}{r} T_{1}^{\ell m}-2 \frac{e^{-2 b}}{r}\left(T_{2}^{\ell m}\right)_{, r} \\
\left.\quad-\left(\frac{\lambda+2}{r^{2}}-\frac{6 e^{-2 b}}{r^{2}}\right) T_{2}^{\ell m}\right\},
\end{aligned}
$$

where $S_{\mathcal{H}}$ and $S_{\chi}$ have been defined in Sec. IC When Eqs. A15 - A17 and the definitions of the coefficients (A3) - A5 are replaced in Eq. (A14), we get $S_{\phi}=\lambda S_{z} / 2$, with $S_{z}$ given by Eq. (21).

The projections of the source stress-energy tensor onto the basis of the spherical harmonics is accomplished as follows. From the orthogonality properties of the harmonics,

$$
\begin{aligned}
& \int d \Omega Y_{\ell m}^{*} Y_{l^{\prime} m^{\prime}}=\delta_{l l^{\prime}} \delta_{m m^{\prime}}, \\
& \int d \Omega Y_{\ell m, a}^{*} Y_{l^{\prime} m^{\prime}, b} \gamma^{a b}=\lambda \delta_{l l^{\prime}} \delta_{m m^{\prime}}, \\
& \int d \Omega Z_{a b}^{* l^{\prime} m^{\prime}} Z_{\ell m}^{a b}=\frac{\lambda(\lambda-2)}{2} \delta_{l l^{\prime}} \delta_{m m^{\prime}}
\end{aligned}
$$



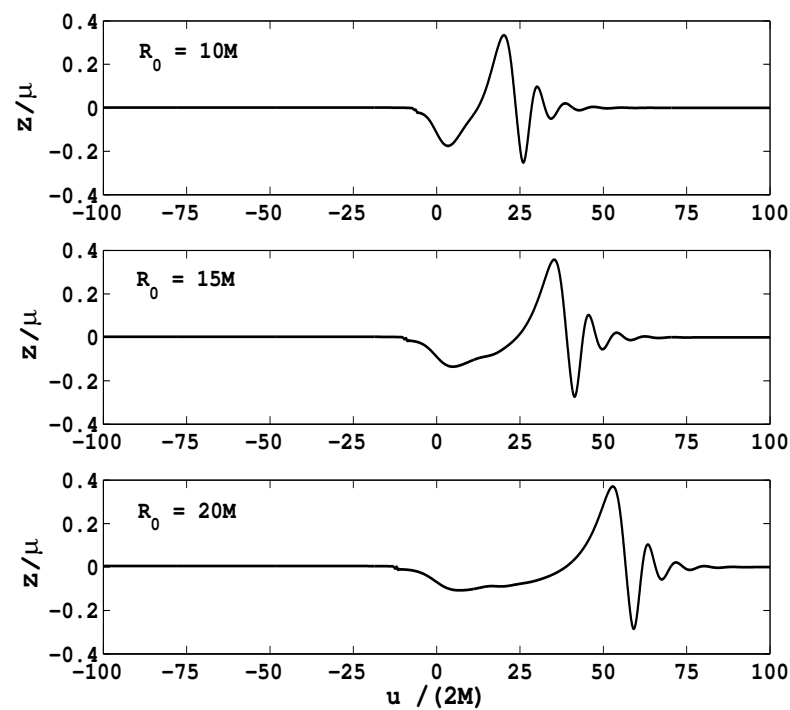

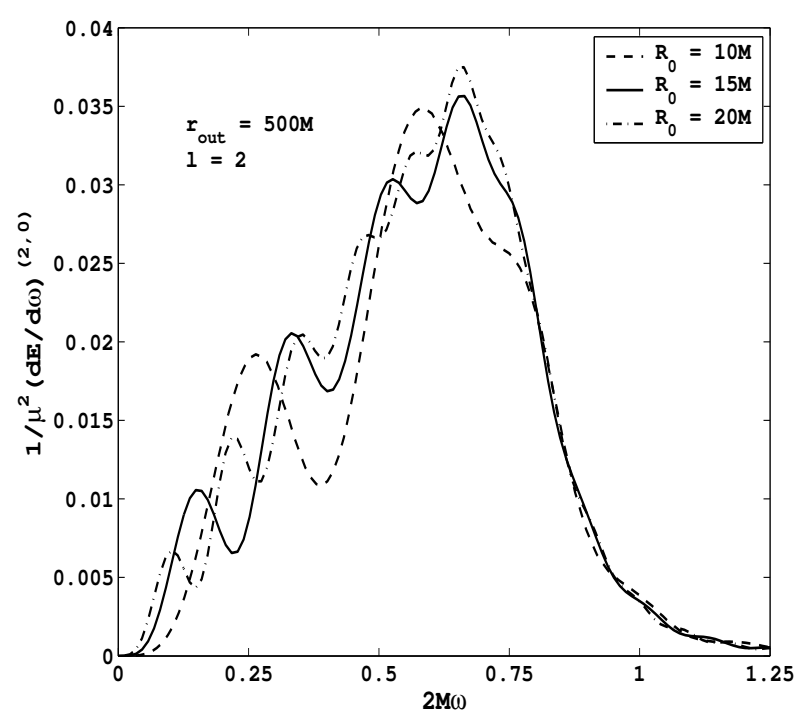

FIG. 11: Gravitational waveforms and energy spectra for a point particle falling radially onto a Schwarzschild black hole from different distances. The left panels show the waveforms and the right panel the corresponding spectral energy distributions. Excellent agreement is found with the results of Lousto and Price [43]. See text for further details.

we obtain the coefficients of the expansion of $t_{\mu \nu}$,

$$
\begin{aligned}
T_{A B}^{\ell m} & =\int d \Omega t_{\mu \nu} Y_{\ell m}^{*}, \quad A, B, \mu, \nu=0,1, \\
T_{0}^{\ell m} & =\frac{1}{\lambda} \int d \Omega\left[t_{02} Y_{\ell m, \vartheta}^{*}-\frac{\mathrm{i} m}{\sin ^{2} \vartheta} t_{03} Y_{\ell m}^{*}\right], \\
T_{1}^{\ell m} & =\frac{1}{\lambda} \int d \Omega\left[t_{12} Y_{\ell m, \vartheta}^{*}-\frac{\mathrm{i} m}{\sin ^{2} \vartheta} t_{13} Y_{\ell m}^{*}\right], \\
T_{3}^{\ell m} & =\frac{1}{2 r^{2}} \int d \Omega\left[t_{22}+\frac{1}{\sin ^{2} \vartheta} t_{33}\right] Y_{\ell m}^{*},
\end{aligned}
$$

and

$$
\begin{aligned}
T_{2}^{\ell m} & =\frac{2}{\lambda(\lambda-2)} \int d \Omega \frac{t_{22}}{2} W_{\ell m}^{*}+\frac{2 t_{23}}{\sin \vartheta} X_{\ell m}^{*} \\
& +\frac{t_{33}}{\sin ^{2} \vartheta}\left[-m^{2} Y_{\ell m}^{*}+\frac{\lambda}{2} \sin ^{2} \vartheta Y_{\ell m}^{*}+\frac{1}{2} \sin 2 \vartheta Y_{\ell m, \vartheta}^{*}\right]
\end{aligned}
$$

where, following Regge and Wheeler [36], we have defined the functions

$$
\begin{aligned}
X_{\ell m}^{*} & =2 \mathrm{i} m\left[\cot \vartheta Y_{\ell m}^{*}-Y_{\ell m, \vartheta}^{*}\right] \\
W_{\ell m}^{*} & =2 Y_{\ell m, \vartheta \vartheta}^{*}+\lambda Y_{\ell m}^{*} .
\end{aligned}
$$

In the general case where $t_{\mu \nu}$ describes a complex source corresponding to a general distribution of matter evolving dynamically, these integrals have to be evaluated numerically. The analytic computation can be done only in some simple cases, such as the particular case when the source is a test-mass body moving along a geodesic of Schwarzschild spacetime [18].

\section{APPENDIX B: POINT-LIKE PARTICLES RADIALLY FALLING ONTO BLACK HOLES.}

In this appendix we reexamine the simplified scenario of a point particle radially falling onto a Schwarzschild black hole. This is done with two purposes: first, to test our perturbative numerical code with previous works and, second, to analyze the similarities and differences with the case of accretion of extended fluid shells onto black holes. The emission generated by infalling particles has been extensively studied in the past. The seminal calculation of the GW emission when a test particle falls from infinity 9] was later extended to non-radial trajectories by Detweiler and Szedenits [50]. In both cases the analysis was done in the frequency domain. A frequency component treatment based on Laplace transforms was also employed by Lousto and Price [43] in the study of the emission from particles falling from finite distances. On the other hand, a treatment of the same problem in the time domain has just recently been approached by Martel and Poisson [38].

In order to test our numerical code we should be able to reproduce those results reported in Refs. [38, 43] within the current time domain approach. A way to deal with a $\delta$-like source and with a discontinuous Zerilli-Moncrief function at the location of the particle was developed by Lousto and Price [4], and later successfully applied in Ref. [38] following a time domain approach. However, we found it convenient and accurate enough for our purposes to represent the $\delta$ function in the particle source terms by a narrow Gaussian written as

$$
\delta\left(r-R_{0}\right) \approx \frac{1}{\sigma \sqrt{2 \pi}} e^{-\left(r-R_{0}\right)^{2} / 2 \sigma^{2}},
$$




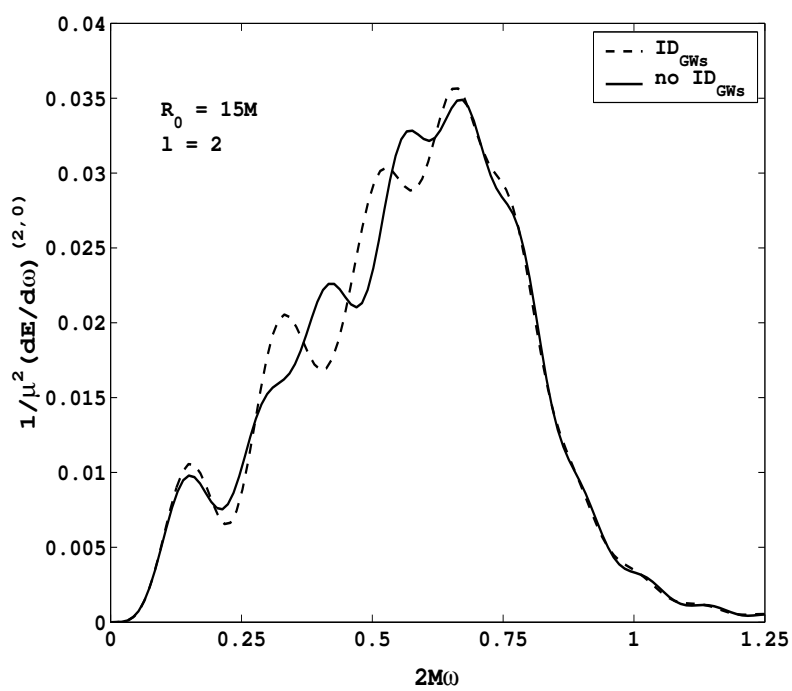

FIG. 12: Effects of the gravitational wave content of the initial data on the energy spectra of a point-like particle falling radially onto a black hole from $R_{0}=15 M$. By eliminating the initial contribution of GWs the amplitude of the bumps is strongly reduced. Qualitatively, however, the effect is always present.

with $\sigma \ll 1$. For a given resolution, we fix $\sigma=2 \Delta r^{*}$, so that the smaller $\Delta r^{*}$, the better the approximation of the $\delta$ function is. The Zerilli-Moncrief equation is solved on an evenly spaced grid using a standard three-level leapfrog scheme. We consider particles initially at rest falling from a finite distance $R_{0}$ to compare with the results of Ref. [43]. Since the particle is falling along the $z$ axis, the system is axisymmetric and the only nonvanishing contribution for any $l$ is the $m=0$ one. The source terms are specified accordingly and we consider explicitly just $l=2$.

As a test of our numerical method, we consider the particle falling from $R_{0}=10 M, 15 M$, and $20 M$, with the same initial setup of Ref. [43] (i.e. including some initial GW content) and compute the evolution of the Zerilli-Moncrief function and its corresponding energy spectrum. Figure 11 shows the results of these simulations. The left panels show the temporal evolution of $Z$ normalized to the particle mass, while the right panel exhibits the corresponding energy spectra. The waveforms show a good agreement in amplitude and shape with those of Ref. [43]. The energy spectra for $R_{0}=10 M$, $15 M$, and $20 M$ must be compared with Figs. 6(b), 4(b), and 4(c) of Ref. 43], respectively. The spectra show the same localized bumps due to the interference between the initial GW pulse and the GWs emitted by the particle during its motion. We notice quite good agreement for $R_{0}=10 M$ and $R_{0}=15 M$, while some small differences are found for $R_{0}=20 M$.

Next, we analyze the contribution of the initial data on the power spectra. We have studied how the spectrum changes when the initial GW contribution is eliminated from the evolution. This is accomplished in the same way we used for the extended shells; that is, the particle is frozen at its location until the initial pulse has gone from the numerical domain, after which the evolution starts. Figure 12 compares the energy spectra emitted by a particle falling from $R_{0}=15 M$ with (dashed line) and without (solid line) the initial GW contribution. The signal is extracted at $r=500 M$ in the two cases. As suggested in a more general scenario in Ref. [38], our results confirm that in the test particle case the bumps in the spectrum are mainly due to the spurious contribution of the radiation in the initial data. In fact, when removing the initial GW content, the amplitude of the bumps is reduced. Some modulation is, nevertheless, still present and the spectrum does not fully correspond to that of a pure QNM ringdown signal. Martel and Poisson [38] argued that this is to be interpreted as an interference effect as well, but between the waves emitted by the particle during its motion and those previously emitted and backscattered by the potential.
[1] S. Chandrasekhar, The Mathematical Theory of Black Holes (Oxford University Press, New York, 1983).

[2] K.D. Kokkotas and B.G. Schmidt, Living Rev. Relativ. 2, 2 (1999).

[3] M. Sasaki and T. Nakamura, Phys. Lett. A 87A, 85 (1981).

[4] M.P. Haugan, S.L. Shapiro, and I. Wasserman, Astrophys. J. 257, 283 (1982).

[5] S.L. Shapiro and I. Wasserman, Astrophys. J., 260, 838 (1982).

[6] K. Oohara, and T. Nakamura, Phys. Lett. A 98A, 407 (1983).

[7] K. Oohara, and T. Nakamura, Progr. Theor. Phys. 70, 757 (1983).

[8] L.I. Petrich, S.L. Shapiro, and I. Wasserman, Astrophys. J. Suppl., 58, 297 (1985).
[9] M. Davis, R. Ruffini, W.H. Press, and R.H. Price, Phys. Rev. Lett. 27, 1466 (1971).

[10] P. Papadopoulos and J.A. Font, Phys. Rev. D 59, 044014 (1999).

[11] S.A. Teukolsky, Phys. Rev. Lett. , 29, 1114 (1972).

[12] J.M. Bardeen and W.H. Press, J. Math. Phys., 14, 7 (1973).

[13] K.D. Kokkotas and B.F. Schutz, Mon. Not. R. Astron. Soc., 255, 119 (1992).

[14] N. Andersson and K.D. Kokkotas, Phys. Rev. Lett. 77, 4134 (1996).

[15] V. Ferrari and K.D. Kokkotas, Phys. Rev. D 62, 107504 (2000).

[16] G. Allen, N. Andersson, K.D. Kokkotas, and B.F. Schutz, Phys. Rev. D 58, 124012 (1998).

[17] J. Ruoff, Phys. Rev. D 63, 064018 (2001). 
[18] J. Ruoff, P. Laguna, and J. Pullin, Phys. Rev. D 63, 064019 (2001).

[19] N. Andersson, K.D. Kokkotas, P. Laguna, P. Papadopoulos, and M.S. Sipior, Phys. Rev. D, 60, 124004 (1999).

[20] G.D. Allen, N. Andersson, K.D. Kokkotas, P. Laguna, J.A. Pullin, and J. Ruoff, Phys. Rev. D 60, 104021 (1998).

[21] E. Seidel and T. Moore, Phys. Rev. D 35, 2287 (1987); E. Seidel, E.S. Myra and T. Moore, ibid. 38, 2349 (1988); E. Seidel, ibid 44, 950 (1991).

[22] E. Seidel, Phys. Rev. D 42, 1884 (1990).

[23] U.H. Gerlach and U.K. Sengupta, Phys. Rev. D 19, 2268 (1979); 22, 1300 (1980).

[24] T. Harada, H. Iguchi, and M. Shibata, Phys. Rev. D 68, 24002 (2003).

[25] J.M. Martín-García and C. Gundlach, Phys. Rev. D 59, 064031 (1999).

[26] C. Gundlach and J.M. Martín-García, Phys. Rev. D 61, 084024 (2000).

[27] J.M. Martín-García and C. Gundlach, Phys. Rev. D 64, 024012 (2001).

[28] F. Siebel, J.A. Font, and P. Papadopoulos, Phys. Rev. D 65, 024021 (2001).

[29] P. Papadopoulos and J.A. Font, Phys. Rev. D 63, 044016 (2001).

[30] F.J. Zerilli, Phys. Rev. D 2, 2141 (1970).

[31] V. Moncrief, Ann. Phys. (N.Y.) 88, 323 (1974).

[32] A. Nagar et al. (in preparation).

[33] L. Lindblom and S.L. Detweiler, Astrophys. J., Suppl. Ser., 53, 73 (1983); S.L. Detweiler and L. Lindblom, Astrophys. J. 292, 12 (1985).
[34] J.A. Pons, E. Berti, L. Gualtieri, G. Miniutti, and V. Ferrari, Phys. Rev. D 65, 104021 (2002).

[35] F. Banyuls, J.A. Font, J.M. Ibáñez, J.M. Martí, and J.A. Miralles, Astrophys. J. 476, 221 (1997).

[36] T. Regge and J.A. Wheeler, Phys. Rev. 108, 1063 (1957).

[37] W. Krivan, P. Laguna, P. Papadopoulos, and N. Andersson Phys. Rev. 56, 3395 (1997).

[38] K. Martel and E. Poisson, Phys. Rev. D 66, 084001 (2001).

[39] P. Papadopoulos and J.A. Font, Phys. Rev. D 58, 024005 (1998).

[40] F.C. Michel, Astrophys. Space Sci. 15, 153 (1972).

[41] E.W. Leaver, Proc. R. Soc. London, A402, 285 (1985).

[42] C.T. Cunningham, R.H. Price, and V. Moncrief, Astrophys. J., 224, 643 (1978).

[43] C.O. Lousto and R.H. Price, Phys. Rev. D 55, 2124 (1997)

[44] C.O. Lousto and R.H. Price, Phys. Rev. D 56, 6439 (1997).

[45] R.H. Price, Phys. Rev. D 5, 2419 (1972); 5, 2439 (1972).

[46] E.S.C. Ching, P.T. Leung, W.M. Suen, and K. Young, Phys. Rev. D 52, 2118 (1995).

[47] H. Dimmelmeier, J.A. Font, and E. Müller, Astron. Astrophys. 393, 523 (2002).

[48] O. Zanotti, L. Rezzolla, and J.A. Font, Mon. Not. R. Astron. Soc. 341, 832 (2003).

[49] J.A. Font and F. Daigne, Astrophys. J. Lett. 581, L23 (2004).

[50] S.L. Detweiler and E. Szedenits, Astrophys. J. 231, 211 (1979). 